\title{
Evaluación de impacto ambiental del sector eléctrico en el norte de México: evolución histórica e implicaciones para la sostenibilidad
}

\author{
María E. GonZÁlez Ávila \\ Luis Felipe Beltrán Morales \\ Julio César Peralta Gallegos \\ ENRIQUe Troyo Diéguez \\ Alfredo Ortega Rubio*
}

\begin{abstract}
We revised and analysed the development and content of the Reportes de Evaluación de Impacto Ambiental - REIA (Evaluation Reports on Environmental Impact) for electricity projects in the last 30 years in Northern Mexico. We also collected and analysed information about the population and the economical situation in the region. We found that neither the process nor the format of the REIA have evolved. The main limitations are: lack of knowledge, ambiguity in the methodological aspects and specific environmental regulations. We recommend the development of REIA specific to the electrical sector, in order to include not only the environmental part but also the public participation and the specific economical aspects of the Northern Mexican region.
\end{abstract}

Keywords: electricity generation, economical growth, environmental impact, sustainable development, Northern Mexico.

\section{Resumen}

Se revisó y analizó el desarrollo y contenido de REIA (Reportes de Evaluación de Impacto Ambiental) de proyectos eléctricos realizados en los últimos 30 años en el norte de México, y se conjuntó y analizó información poblacional y económica de la zona. Encontramos que tanto el proceso como los formatos de los REIA no han evolucionado. Las principales limitaciones son: falta de conocimiento y ambigüedad en aspectos metodológicos, y regulaciones ambientales específicas. Recomendamos el desarrollo de REIA específicos del sector eléctrico que involucren no sólo la parte ambiental, sino además la participación pública y los aspectos económicos específicos de la región norte de México.

Palabras clave: generación de electricidad, crecimiento económico, impacto ambiental, desarrollo sostenible, norte de México.

*Centro de Investigaciones Biológicas del Noroeste, La Paz, Baja California Sur. Correos-e: megamar04@cibnor.mx, lbeltran04@cibnor.mx, cperalta04@cibnor.mx, etroyo04@cibnor.mx, autor corresponsal: aortega@cibnor.mx. 


\section{Introducción $^{1}$}

Durante la primera mitad del siglo pasado, las principales inversiones en el sector eléctrico mexicano provenían de compañías extranjeras. En 1933, el gobierno mexicano creó la Comisión Federal de Electricidad (CFE), y durante 1963 se formó la Compañía de Luz y Fuerza del Centro, S.A. (ClyfC). Ambas compañías fueron y son las principales generadoras y distribuidoras de electricidad en México (ввив, 2002). Los requerimientos de energía eléctrica han aumentado mas rápidamente que el producto interno bruto (PIB) del país, por eso resulta necesario que en los próximos años la CFE y la CLyFC incrementen de manera significativa la producción eléctrica. Para ello, el gobierno ha considerado una inversión de mas de cinco mil millones de dólares anuales (вBVB, 2002).

Además de lo anterior se hace necesario un incremento en la competitividad, productividad y eficiencia del sector eléctrico, para un verdadero desarrollo social y económico que al mismo tiempo ofrezca formas limpias y eficientes de producción de electricidad, disminución de los impactos negativos e incremento de la calidad de vida de las poblaciones locales y regionales (Belausteguigoitia et al., 2001), y, por supuesto, se requiere incrementar el número de centrales eléctricas actuales y modernizar y dar mantenimiento a las centrales ya existentes, así como incrementar su capacidad y apoyar el desarrollo de energía no convencional como la eólica y la solar. Desde el punto de vista ambiental es necesario desarrollar eficientes Reportes de Evaluación de Impacto Ambiental (REIA), lo cual contribuirá decisivamente en una producción de electricidad limpia y ambientalmente amigable. En ese contexto, en el presente trabajo se examina la historia de los REIA, su aplicación en las entidades federativas del norte de México (Baja California, Baja California Sur, Coahuila, Durango, Nuevo León, Sinaloa, Sonora y Tamaulipas), y las propuestas para su mejoramiento y optimación.

\footnotetext{
${ }^{1}$ Este estudio fue apoyado por Centro de Investigaciones Biológicas del Noroeste y por el Conacyt-Semarnat, Project 2002-C01-0844. Agradecemos a la Dra. Rosario Rogel Salazar y a dos revisores anónimos por sus valiosos comentarios y sugerencias para mejorar las versiones preliminares de este trabajo.
} 


\section{Materiales y métodos}

La presente investigación incluyó la búsqueda, recopilación y análisis de información bibliográfica y vía internet, así como la consulta con expertos y el minucioso análisis de todos los diferentes tipos de REIA en la Secretaría de Medio Ambiente y Recursos Naturales (Semarnat), de proyectos termoeléctricos, hidroeléctricos, geoeléctricos y eólicos desarrollados en el norte de México. Se analizaron asimismo las estadísticas poblacionales, económicas y energéticas publicadas por el Instituto Nacional de Estadística, Geografía e Informática (INEGI, 2002, 2003, 2005a, b y c) y el Consejo Nacional de Población (Conapo, 2000a y b).

\section{Historia del sector eléctrico en México}

La red mexicana de electricidad surgió en 1879, cuando en León, Guanajuato, se instaló la compañía Las Ameritas con varias plantas eléctricas. Para 1881, la compañía Knight instaló las primeras lámparas incandescentes en la Ciudad de México; más tarde, en 1889 se activaron las primeras plantas hidroeléctricas en Batopilas, Chihuahua. En 1895 se concesionó un río en Puebla para producir electricidad (Sener, 1999). Para 1900, la capacidad eléctrica instalada en el país era de 22.3 mil kW, 44\% de lo cual era consumido por las plantas textiles de Veracruz, Nuevo León y Puebla (Sener 1999).

Entre 1887 y 1911 surgieron en México 199 compañías que producían y distribuían 112 mil kW, pero al carecer de regulación resultaron en un monopolio regional, con un servicio caótico y precios altos para el consumidor. En esos años, el presidente Álvaro Obregón reorganizó el sector eléctrico y creó la Comisión Federal de Electricidad (CFE) en 1937. En su primera etapa, la CFE se dio a la tarea de construir plantas generadoras para satisfacer la demanda existente y la de los primeros proyectos en el norte del país: Ures y Altar, en Sonora. Para 1938, la CFE tenía una capacidad de $64 \mathrm{~kW}$; ocho años más tarde aumentó a 45,594 kW (Sener, 1999; IfAI, 2005). Posteriormente, Adolfo López Mateos (1960) nacionalizó la industria eléctrica y compró la Compañía de Luz y Fuerza del Centro (Sener, 1999). En esa década, la generación era de alrededor de 2,308 MW de capacidad instalada en el país, donde la CFE aportaba 54\%; Mexican Light, 25\%; American y Foreign, 12\%, y el resto de las compañías, 9\%. Sin embargo, y a pesar de los esfuerzos de generación 
y electrificación, sólo 44\% de la población contaba con electricidad. A partir de la creación de la CFE, la población creció en 91\% (34.9 millones de habitantes), lo que se conjugó con un acelerado desarrollo de la industria, la agricultura y otras actividades urbanas y rurales (CFE, 2005). Entre 1960 y 1976 se volvió a incrementar la producción de electricidad, que pasó de 1,257 a 10,617 Mw (Rodríguez, 1999: 413-428, 434-439). No obstante, en la década de los ochenta se desaceleró ese crecimiento debido principalmente a falta de asignación de recursos económicos a la generación de energía eléctrica, escasez relacionada básicamente con cambios políticos y económicos nacionales y mundiales.

En lo político, por ejemplo, entre 1960 y 1992 la CFE controló el total del sector, y hacia 1972 ya ejercía un monopolio e inició una expansión fortuita de su infraestructura eléctrica, tanto de generación, transmisión, distribución e integración del sistema de interconexión como de todos los sistemas aislados, a excepción de la península de Baja California, que se mantenía independiente. También modificó ciclos y frecuencias eléctricas de todos sus sistemas y cubrió 95\% del servicio eléctrico de la población total, lo cual indudablemente se debió a la política de expansión e inversión que se dio por el crecimiento de la demanda (Sánchez et al., 2005; Breceda, 2002). En la década de los noventa hubo un parteaguas en el sector eléctrico nacional y mundial, ya que se inició el desarrollo tecnológico del ciclo combinado que emplea gas natural-combustible, que permite procesos más limpios y ambientalmente más amigables, en comparación con el uso de combustóleo o carbón, que resultaban no tan eficientes en la generación de energía eléctrica (Sánchez et al., 2005; Sener, 2003). En el caso de México, después de 1971 hubo un crecimiento de la producción eléctrica de origen termoeléctrico, que pasó de 27 (1937) a 51\% (1971), esto debido al auge petrolero de los setenta, que incentivó el desarrollo de centrales termoeléctricas en zonas urbano-industriales, proceso que se acentuó en los noventa. Además del incremento de centrales termoeléctricas también aumentó el número de las hidroeléctricas, que van asociadas a los cambios tecnológicos, económicos y políticos mundiales de la globalización y que traen como consecuencia una presión para los países en vías de desarrollo como México.

En 1992, México renegoció su deuda y adoptó una política económica de corte neoliberal que implicó que el sector eléctrico creciera a costa del mercado internacional de capital y de 
financiamiento privado (Campos y Quintanilla, 1997). Así, el gobierno procedió a reducir la inversión en todos los aspectos del sector eléctrico y permitió la inversión privada nacional y extranjera, que actualmente es la que vende energía a la CFE y que se ampara en el Tratado de Libre Comercio de América del Norte (TLCAN) (Ángeles, 2003). Tanto los aspectos económicos como los políticos, sociales e históricos están obligando al gobierno mexicano a invertir en este sector, no sólo para ofrecer un buen servicio al consumidor, sino también para proveer electricidad a las comunidades rurales, para exportar electricidad a California, en Estados Unidos, y para reestructurar en términos generales el sector eléctrico. En el mapa i se muestran los principales proyectos eléctricos establecidos en el norte de México (Sener, 2003: 36-37, 59, 74-75, 98-99), y en el cuadro 1 se presentan las características técnicas de las centrales eléctricas construidas en esa región (véase mapa I y cuadro 1).

\section{Desarrollo de los reportes de Evaluación de Impacto Ambiental (EIA)}

Malcolm Hollick (1981: 79) define la EIA como un proceso que tiene como objetivo considerar los factores ambientales en la toma de decisiones en una proporción adecuada. La EIA, en teoría, es una actividad interdisciplinaria que requiere un análisis comprensible y que con una participación propositiva puede derivar en un proyecto bien evaluado (Bojorquez y García, 1998: 217). En los años setenta se desarrolló en Estados Unidos de América el procedimiento de la EIA (Environmental Impact Assessment), introducido por el marco legal de la Ley Nacional de Política Ambiental (National Environmental Policy Act, NEPA) que se promulgó en 1970 (INE, 2000: 43). En esa misma época, en otros países también se inició el proceso de legislación ambiental y el establecimiento de la EIA; tal es el caso de la antigua Unión Soviética, que en 1977 materializó el proceso de la EIA y el establecimiento de instituciones ambientales (Cherp y Lee, 1977: 177-179). En México, la EIA se aplicó hacia 1977, pero sólo en la administración pública federal, y fue hasta la década de los ochenta cuando se inició como un proceso con carácter voluntario e institucional (INE, 2000: 34). Otros países como Indonesia y Brasil incorporaron el procedimiento EIA hasta en 1986 (Purnama, 2003: 413), al igual que la legislación para evaluación ambiental (Glasson y Nemesio-Neves, 2000: 191-208, 218-225), y en el 


\section{Mapa I \\ Ubicación de las principales centrales eléctricas del norte de México}

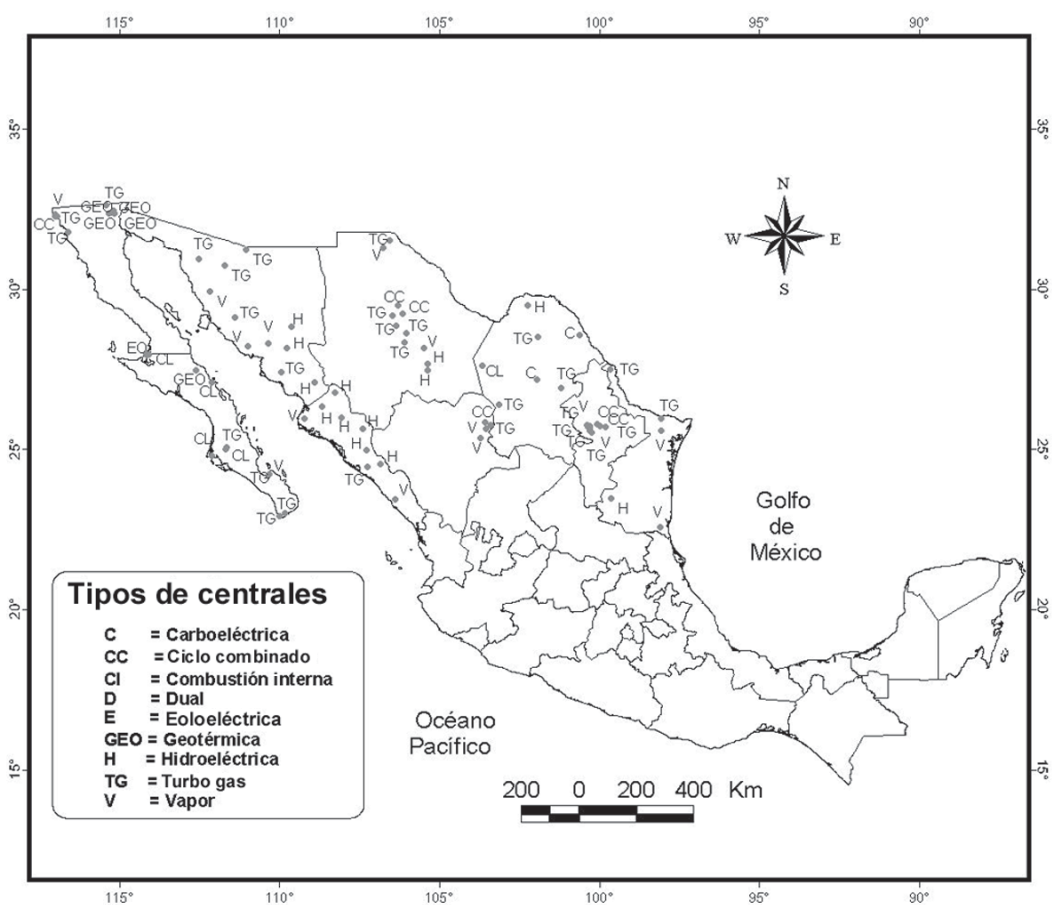

Fuente: Elaboración propia en el Laboratorio de Sistemas de Información Georreferenciada (Labsig)-Cibnor.

caso del Líbano fue hasta 1993 cuando se creó el ministerio ambiental que regula la EIA (El-Fadel et al., 2000: 579).

En México, al igual que en otros países del mundo, es necesario informar a la autoridad ambiental de las actividades y efectos que puede producir determinado proyecto, proceso que se realiza mediante un Reporte de Evaluación de Impacto Ambiental (REIA; en inglés, EIs, Environmental Impact Statement), el cual es sometido a la evaluación de la autoridad y puesto a escrutinio público (Bojorquez y García, 1998: 237). Sin embargo, autores como Exequiel Ezcurra (1995: 1), Julieta Pisanty-Levy (1993: 267) y el propio Instituto Nacional de Ecología (INE, 1994: 1) consideran que la EIA es un proceso ligado exclusivamente a la burocracia. Inicialmente, en nuestro país los REIA se consideraban como un instrumento marginal, que se veía reflejado en el numero de proyectos evaluados: 100 proyectos en toda la década de los ochenta (INE, 2000: 43). Fue hasta 1988 cuando se pu- 


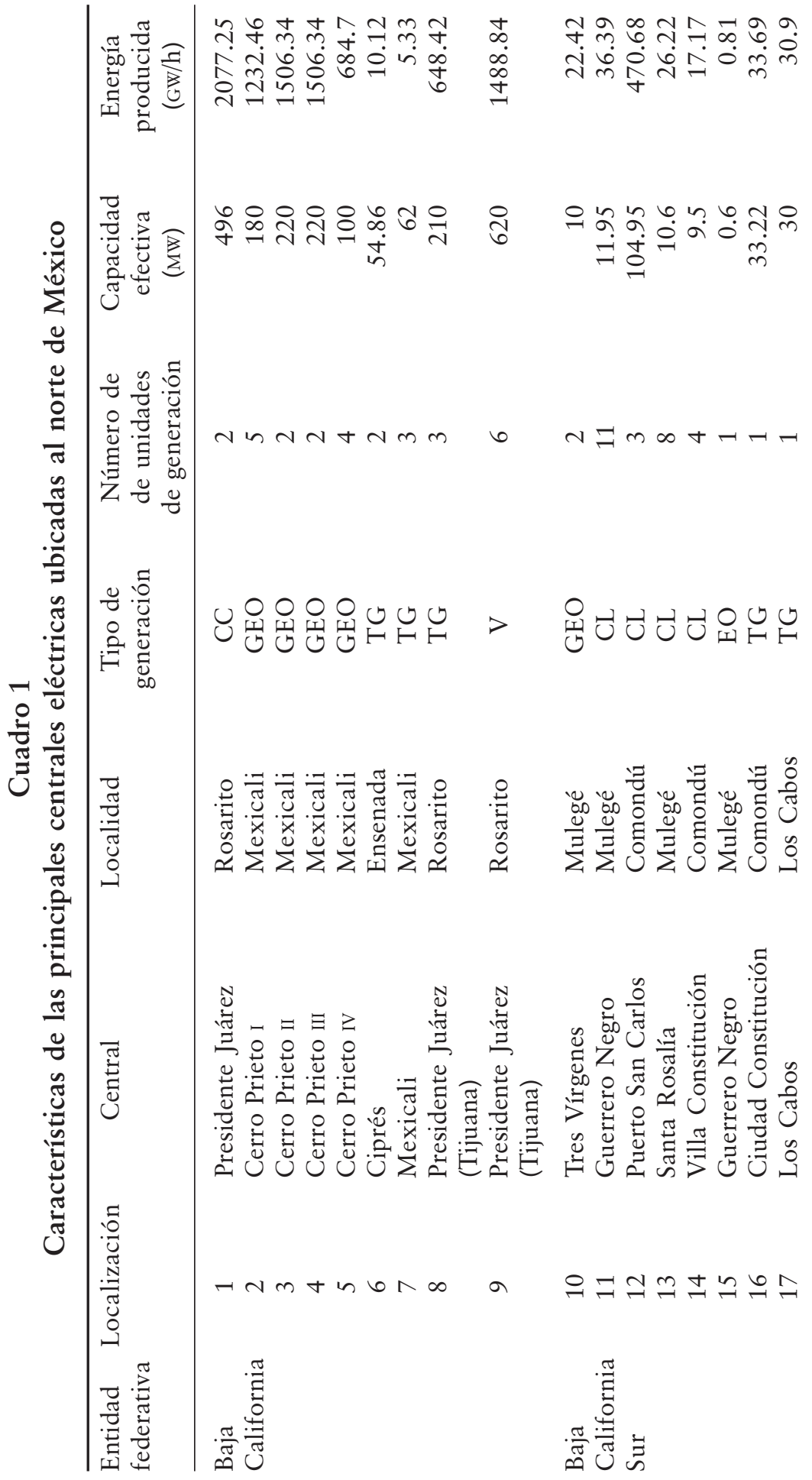




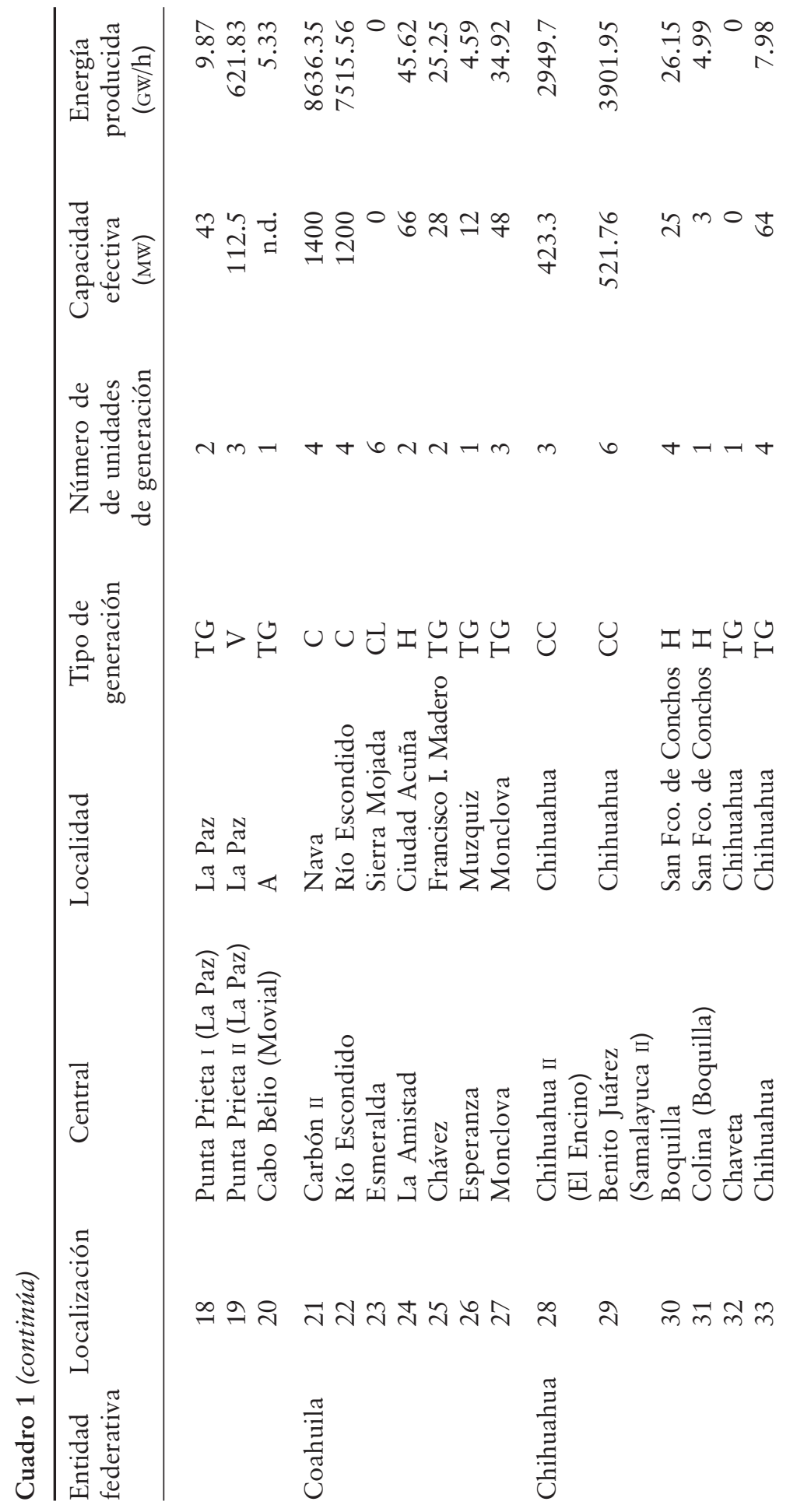




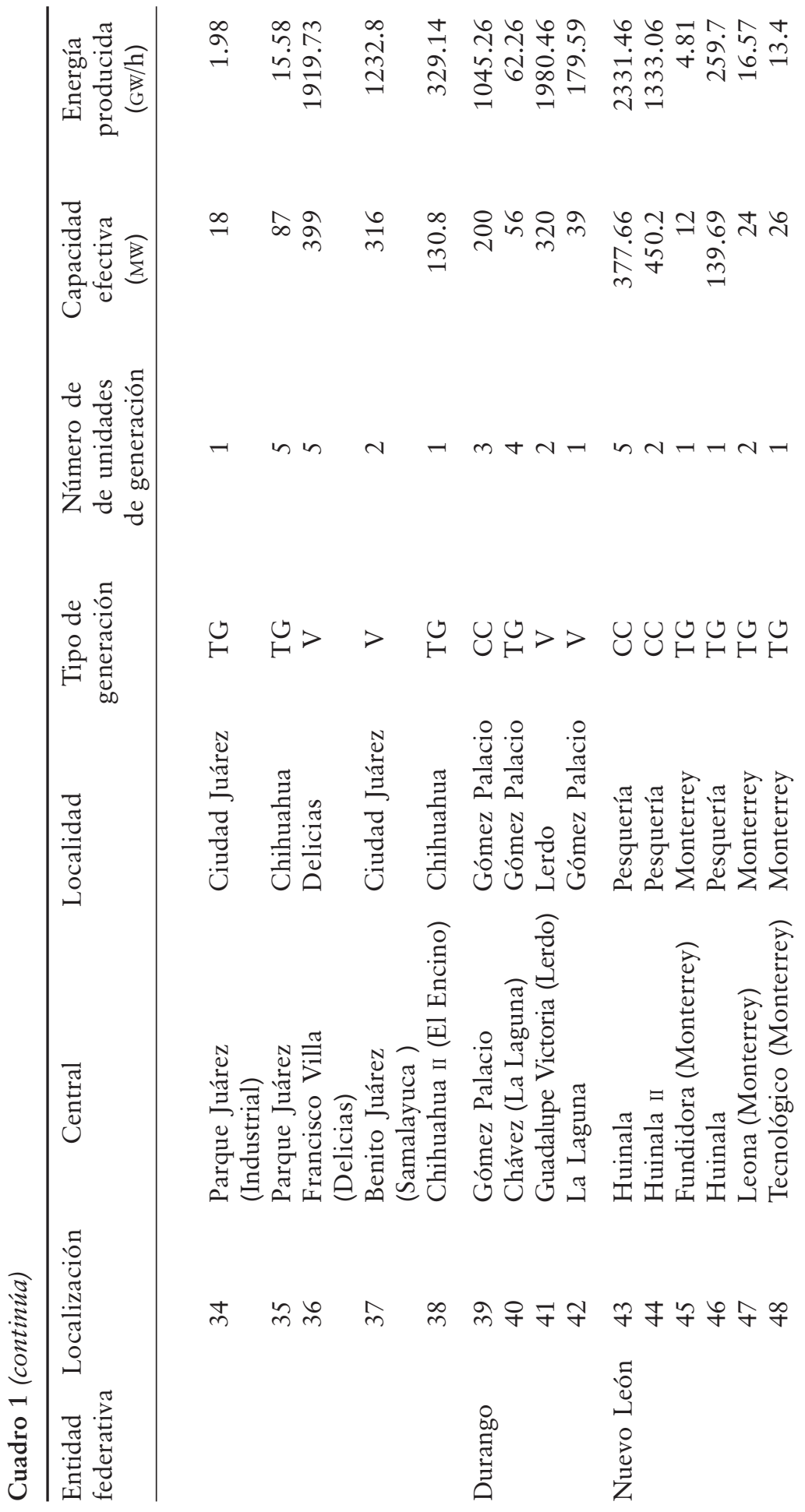




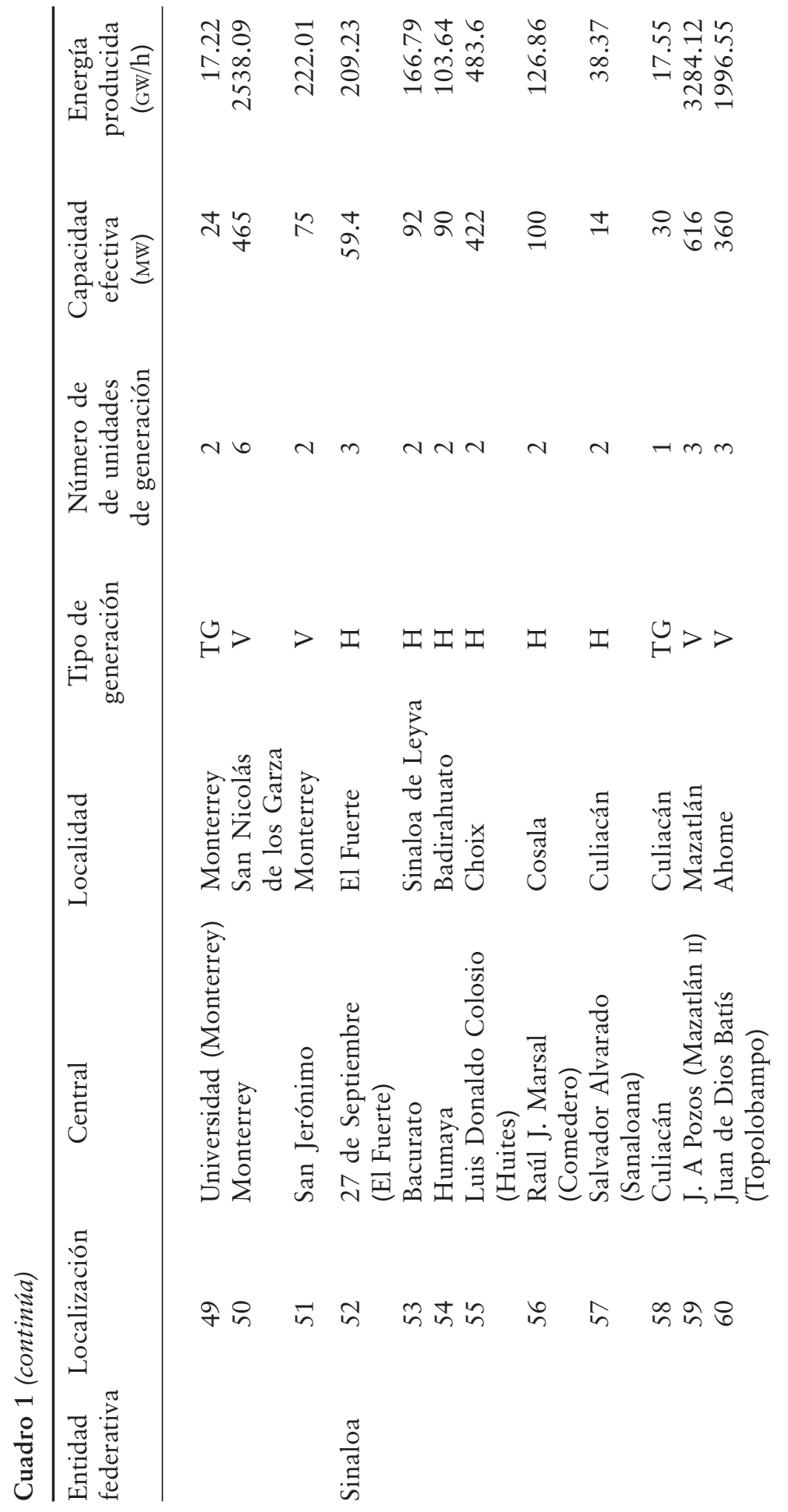




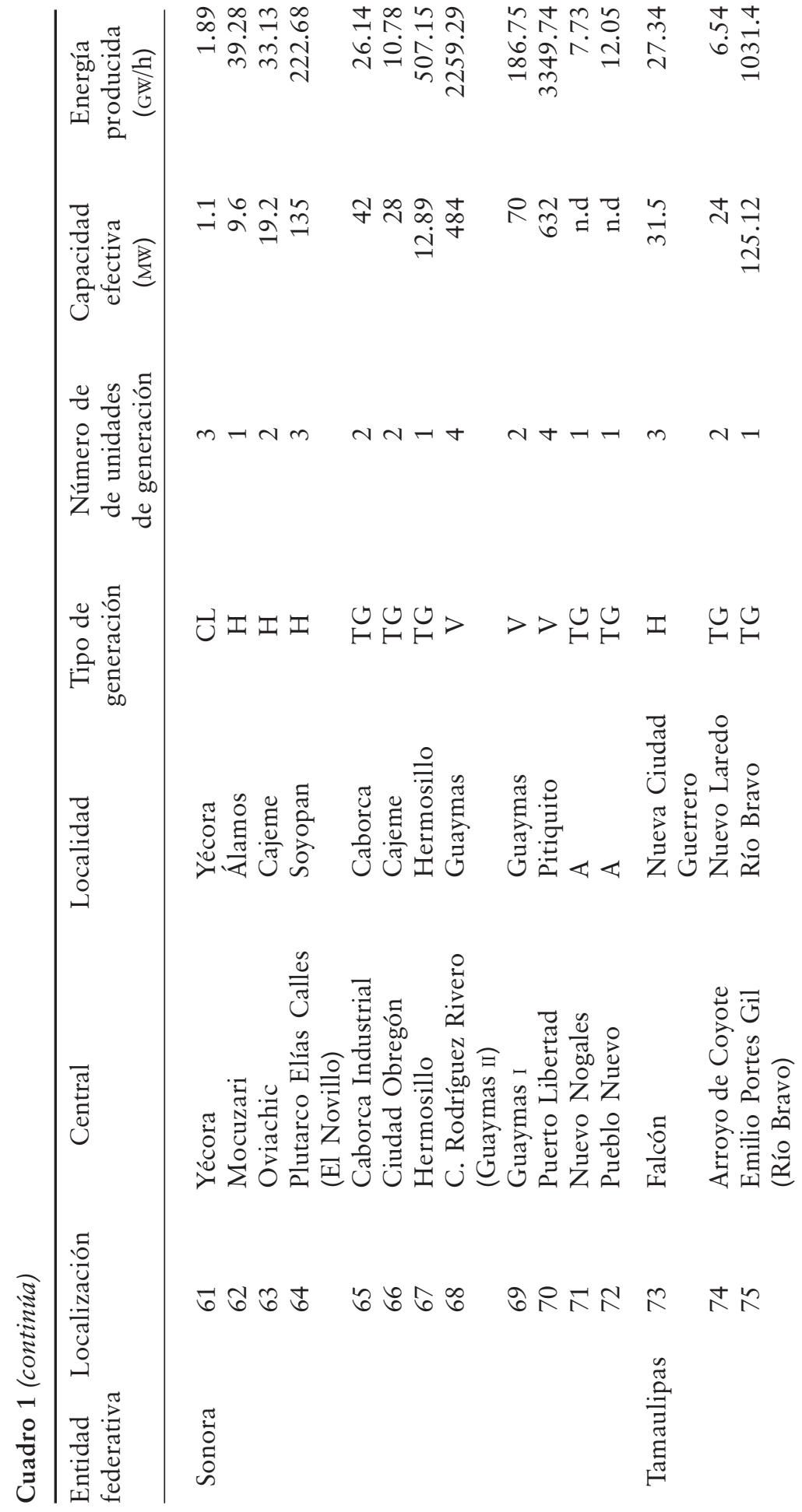




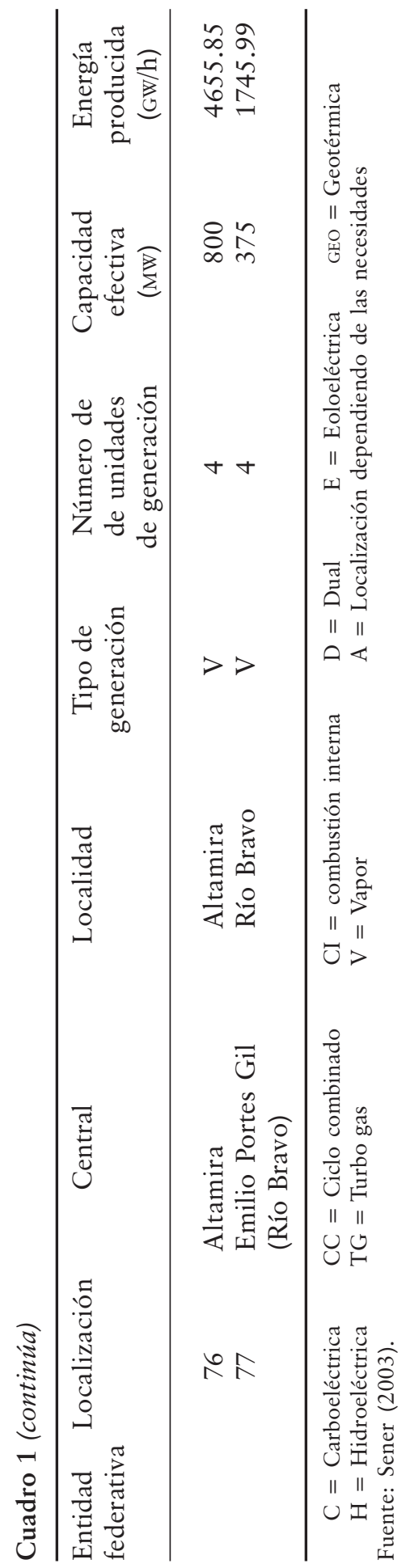


blicó de manera oficial el proceso de evaluación de impacto ambiental, la Ley General del Equilibrio Ecológico y la Protección al Ambiente (LGEEPA), su reglamento (RLGEEPA), así como las normas ambientales aplicables, que son instrumentos útiles durante el proceso de la EIA (INE, 2000: 34).

El desarrollo de la EIA y los REIA en México tiene mucho que ver con la evolución tanto del marco jurídico como del institucional-ambiental para evaluar los posibles impactos ambientales de una obra o proyecto. En la gráfica i se muestra la evolución de la institución ambiental, del marco jurídico y del formato de REIA desde 1980 hasta el 2000 (véase gráfica I). Los REIA aplicados al sector eléctrico mexicano fueron en sus inicios de carácter académico; ejemplo de ello es la tesis de Pisanty-Levy (1976: 12), quien evaluó el impacto ambiental ocasionado por la planta-núcleo eléctrica Laguna Verde, en Veracruz. En el caso de estudios concretos de la zona norte del país se encontraron reportes de la termoeléctrica Lerdo, en Durango (UNAM, 1988: 194-229); de Puerto San Carlos, en Baja California Sur (Cibnor, 1990: 1, 132), y de Samalayuca, en Chihuahua (Ecoprotección Ambiental, s.A. de C.v., 1993: 1-81). En el cuadro 2 se resumen los REIA realizados de 1988 a 2000 para proyectos eléctricos del norte de México (véase cuadro 2). Cabe mencionar que en algunos países de América Latina y el Caribe se aplican REIA muy semejantes a los mexicanos; la excepción son Ecuador y Cuba (Hurtado, 1999: 5), países que desde 1994 cuentan con una guía específica para evaluar tipos de proyectos eléctricos como termoeléctricas, hidroeléctricas y geoeléctricas (Olade-BID, 1994: 13-25; BID, 2001: 4).

\section{Análisis de la información y discusión}

El análisis se realizó en dos etapas: en la primera analizamos los pros y los contras del proceso de la EIA y de los REIA, mientras que en la segunda se trató la vinculación de los REIA con el sector eléctrico de la región norte de México, en virtud de la importancia social, económica y ambiental de esa zona.

\subsection{Reporte de Evaluación Ambiental (REIA)}

En el cuadro 3 se muestran los cambios que se han dado en el procedimiento de la EIA y los tipos de REIA a partir de la modificación del RLGEEPA (véase cuadro 3). Lo que se transformó de manera más significativa fueron las modalidades de los REIA: General, 


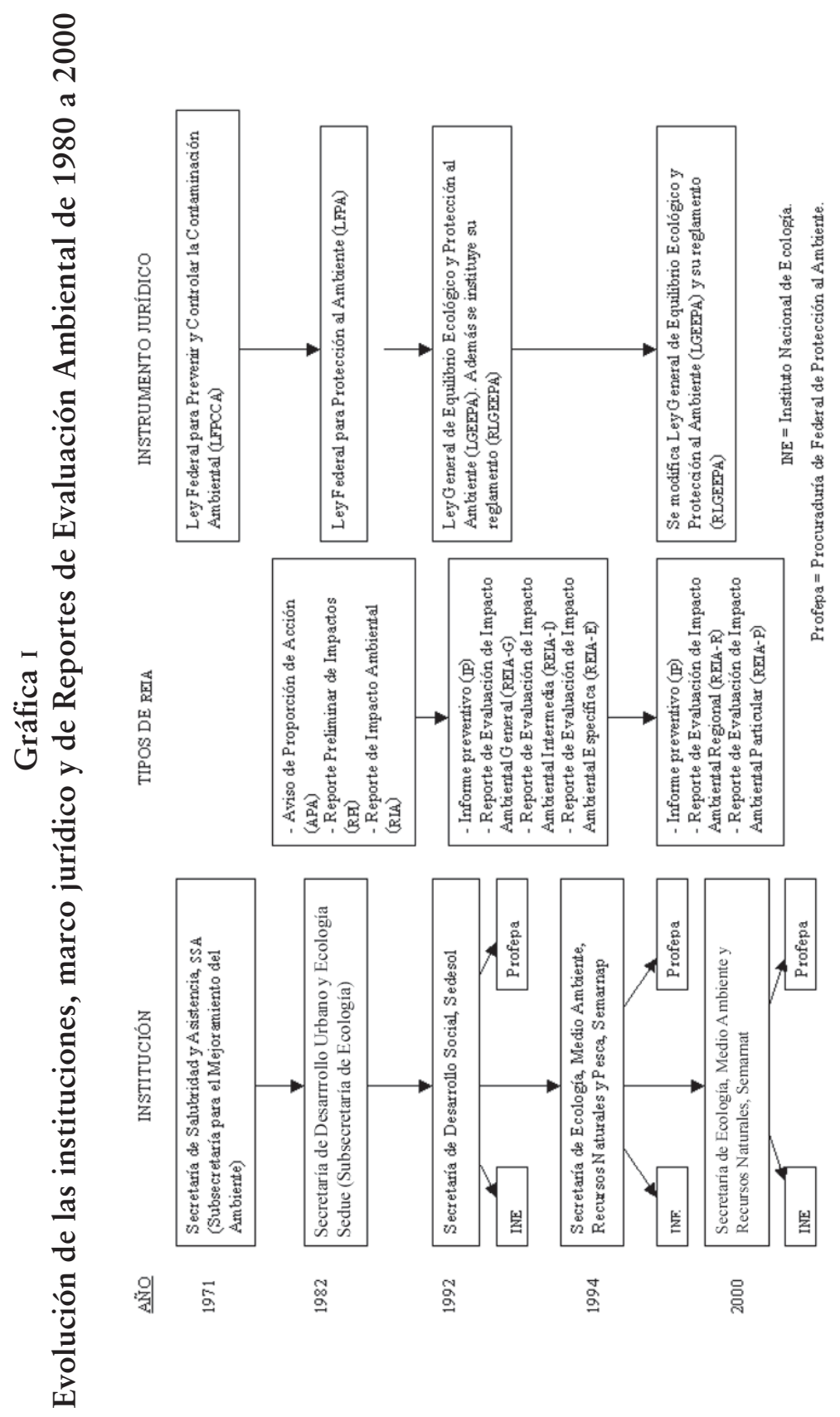

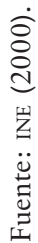




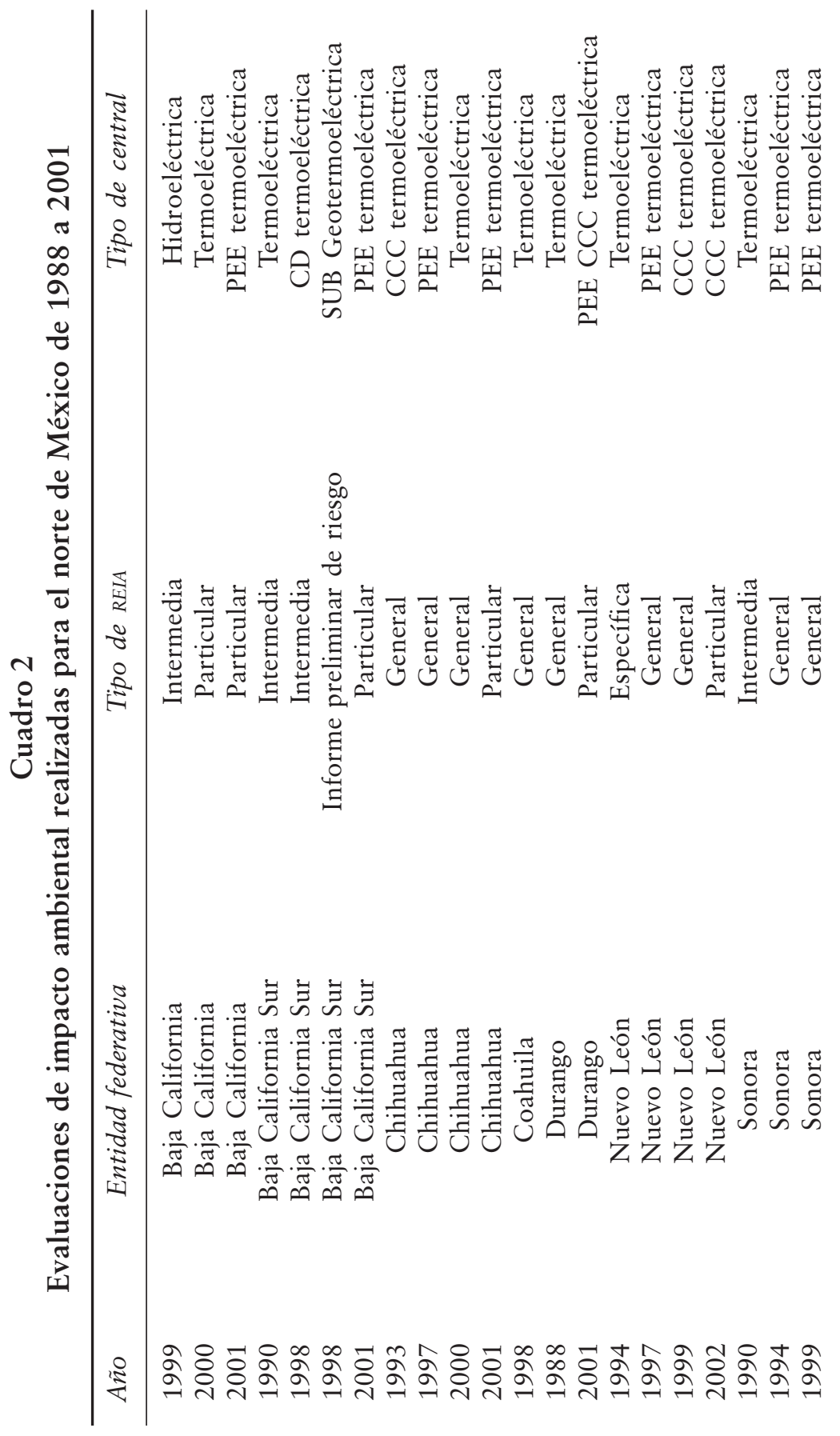




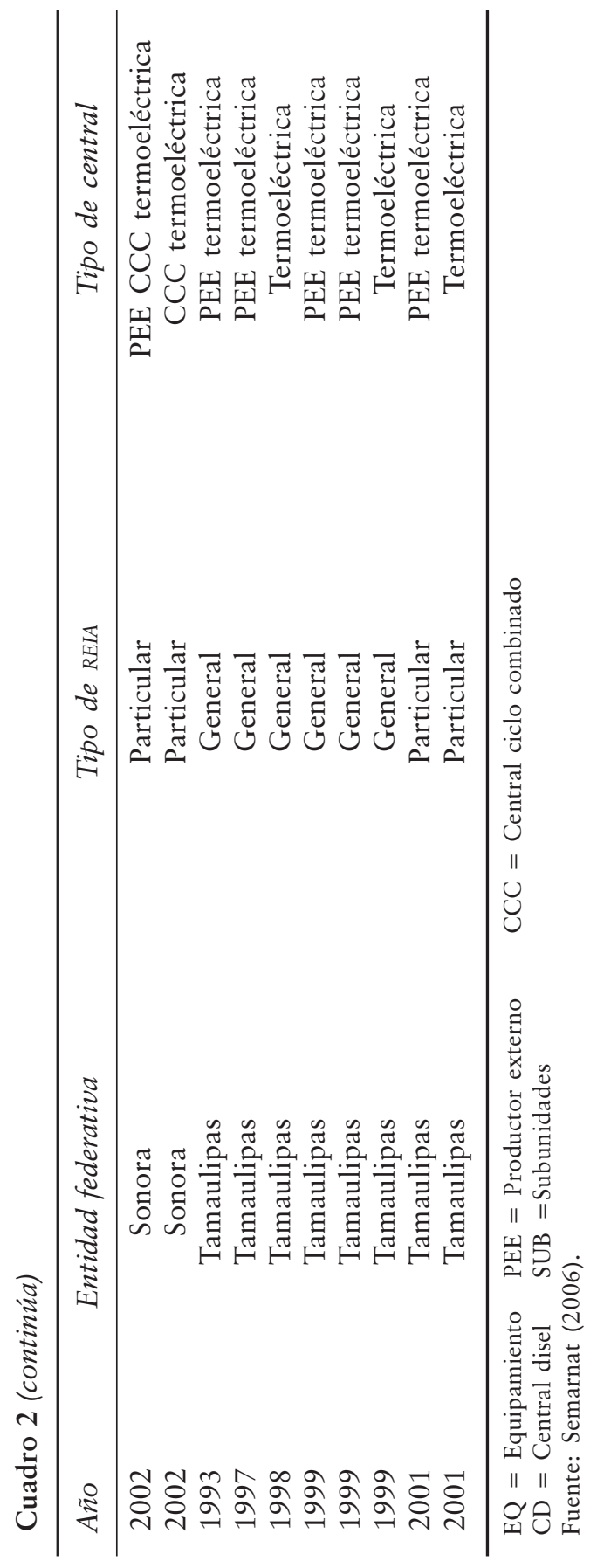


Intermedia y Específica, que cambiaron a sus equivalentes Regional y Particular, lo cual aparentemente agilizó el proceso de evaluación y lo oficializó mediante la publicación de las guías o reportes de evaluación respectivos, que proporciona la Semarnat. En el RLGEEPA no se menciona que el promovente ${ }^{2}$ tenga el deber o la obligación de hacer uso de tales guías o REIA, pero si no se usan se incrementa la posibilidad de que el proyecto no sea aceptado o aprobado, según lo indica Herrera (2003: 227).

Un avance en el proceso y formato de los REIA es la aparición de criterios y tiempos para la revisión de REIA e Informe Preventivo (IP) (Gaceta Ecológica, 1989a), aunque dichos criterios aun son subjetivos, pero aunados a la creación de un expediente para cada tipo de proyecto, se ha dado mayor claridad y transparencia en el proceso de evaluación. Adicionalmente ya se solicita al promovente una garantía, que tiene como finalidad resarcir los potenciales daños ambientales causados en alguna etapa del proyecto; desafortunadamente no se han establecido montos específicos o algún tipo de tabulador que indique la cantidad a pagar por tal o cual daño ambiental.

Otro punto a favorable es la reglamentación para la participación y consulta pública del REIA, lo cual da la oportunidad a una comunidad de manifestar sus inconformidades o sugerir medidas de prevención y mitigación, o en su defecto presentar un REIA que sugiera aspectos no considerados en la primera evaluación. Este avance, sin embargo, resulta contradictorio y poco aplicable ya que: a) legalmente, el promovente y la propia Semarnat tienen el derecho de reservarse la información del REIA cuando el primero lo solicite, o cuando el proyecto sea prioritario para el país; $b$ ) el acceso a la información pública y la participación son limitados por desconocimiento del proceso o forma de consulta, y c) si la comunidad elaborara un REIA tendría en primer lugar que presentar la inconformidad en los tiempos establecidos por la Semarnat, que son cortos por cierto. En segundo lugar, al no tenerse acceso a la información, por el derecho que tiene el promovente a reservarse información, tendría que generar o recopilar su propia información y por supuesto contar con los recursos económicos, técnicos y profesionales para elaborar el documento.

\footnotetext{
2 Promovente: Persona moral o física que somete a evaluación de la secretaría un Informe Preventivo o una Manifestación de Impacto Ambiental (INE, Glosario: http:// www.ine.gob.mx/ueajei/publicaciones/libros/265/glosario.html).
} 


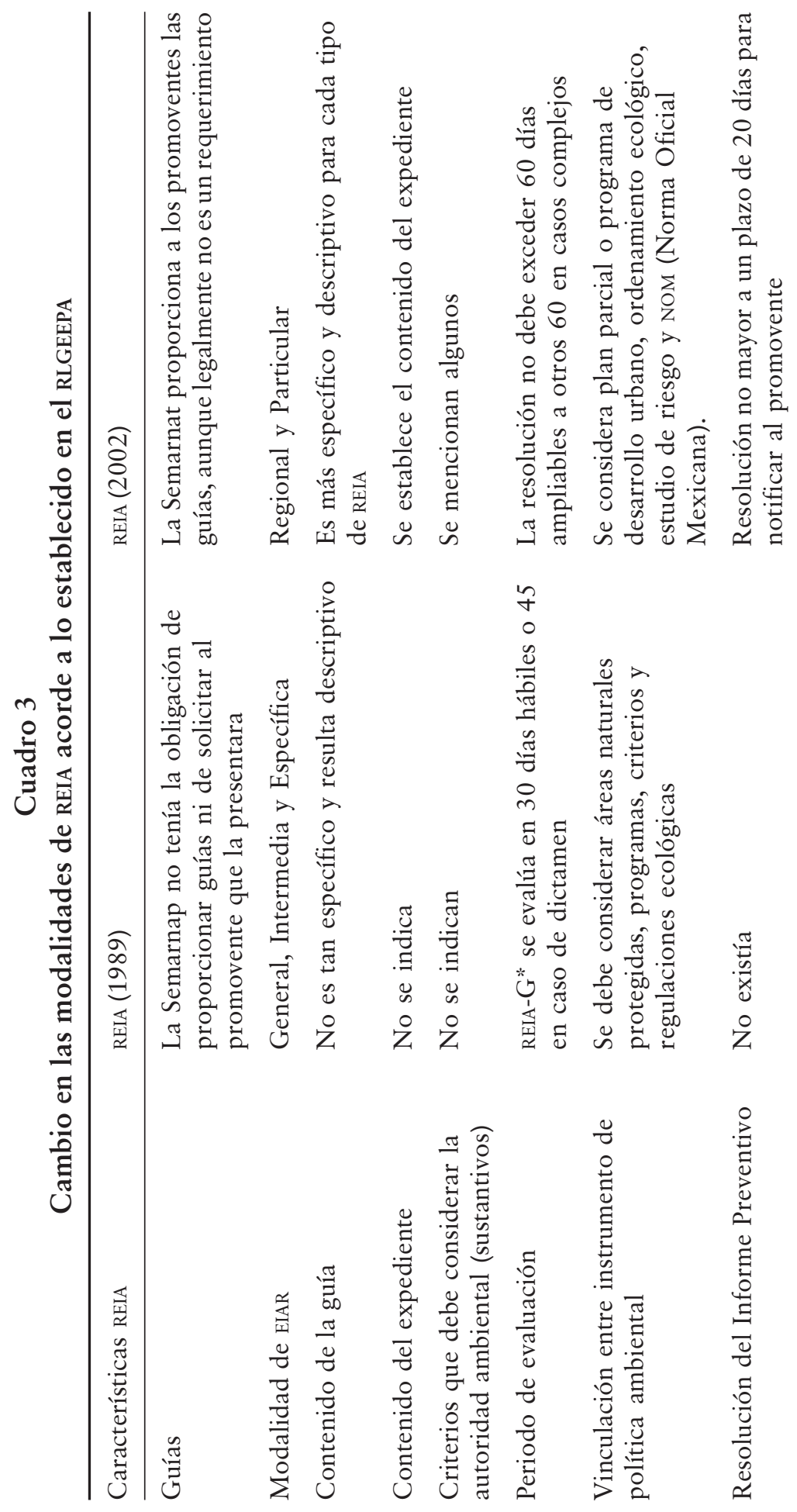




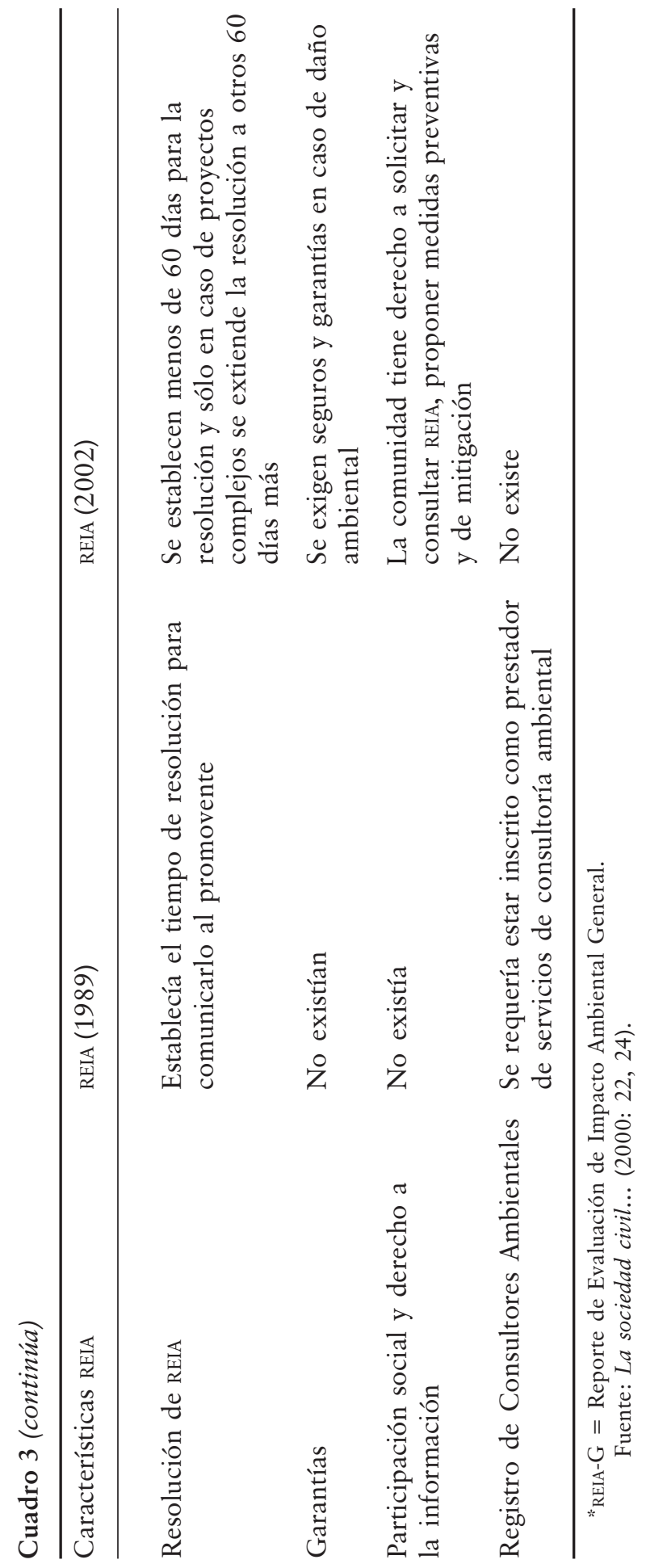


Un punto que para algunos sectores constituyó un avance es la desaparición de la lista de consultores ambientales, ya que anteriormente la Semarnat era juez y parte de la certificación de los consultores. Sin embargo, esto ha limitado al promovente su conocimiento para elegir asesoría especializada para elaborar REIA, por lo que se sugiere crear un registro de los consultores, que indique su especialidad y calidad profesional, y éstos podrían estar certificados por un comité de especialistas y académicos de área en cuestión.

En el cuadro 4 se hace un comparativo de los principales puntos contenidos en los REIA actuales y pasados. Por ejemplo, en apartado III de las nuevas modalidades de REIA se observa la incorporación de instrumentos de planeación y ordenamientos jurídicos aplicables al proyecto, lo cual en los REIA anteriores se consideraba superficialmente (apartado IV, REIA General e Intermedia). Cuando se realizo la revisión de reglamentos y normas aplicadas en diferentes proyectos y actividades del sector eléctrico, se observó que no existe una homogeneidad en cuanto a instrumentos $\mathrm{u}$ ordenamientos jurídicos para ese sector, por lo que el apartado resulta sólo un listado de reglamentos y normas que pueden o no aplicarse. Se sugiere que la Semarnat y la CFE, así como investigadores y consultores, establezcan las normas y regulaciones aplicables al sector eléctrico por tipo de proyecto, lo que facilitará su seguimiento durante las supervisiones y auditorías que realiza la Procuraduría Federal de Protección al Ambiente (Profepa).

En el apartado v, las nuevas modalidades de REIA consideran la identificación y evaluación de impactos acumulativos y residuales, que anteriormente no se tomaban en cuenta. El apartado VI resulta semejante para todos los tipos de REIA; la novedad son las especificaciones de prevención y mitigación de impactos acumulativos y residuales, aunque no consideran escenarios posibles sino hasta el apartado VII. Finalmente, el apartado VIII de las nuevas modalidades de REIA involucra un mayor número de instrumentos metodológicos y elementos técnicos para sustentar la evaluación (véase cuadro 5), aunque en la práctica su uso y aplicación para la identificación, evaluación y propuestas de medidas de mitigación es limitado, tanto por desconocimiento teórico y práctico de estos métodos, como porque en algunos casos dichos métodos son desconocidos o están en desuso.

La revisión de los proyectos eléctricos evidenció que se sigue usando la matriz de Leopold (1971). Al analizar 51 REIA de proyectos eléctricos se observó que 36 REIA de proyectos termoeléc- 


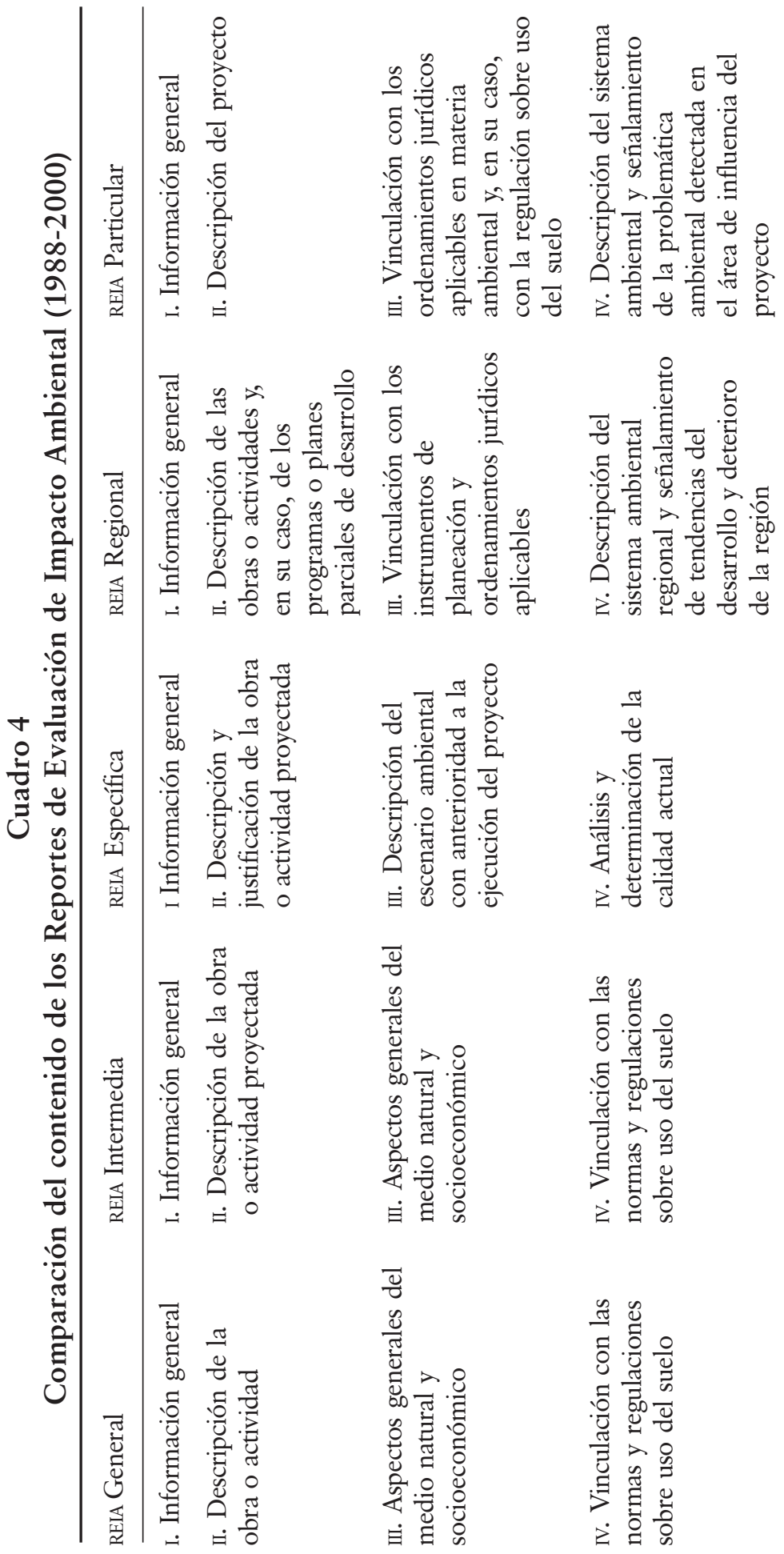




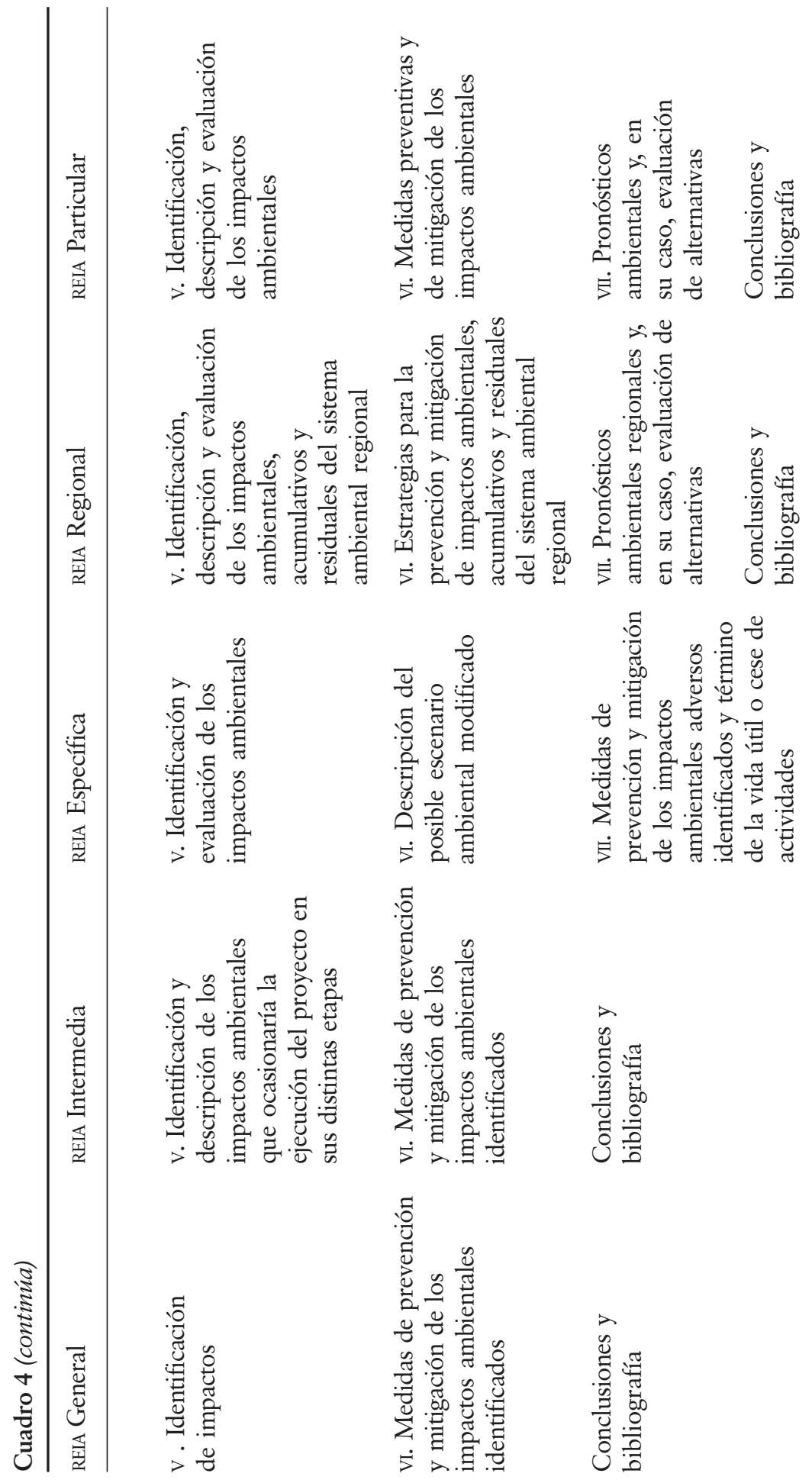




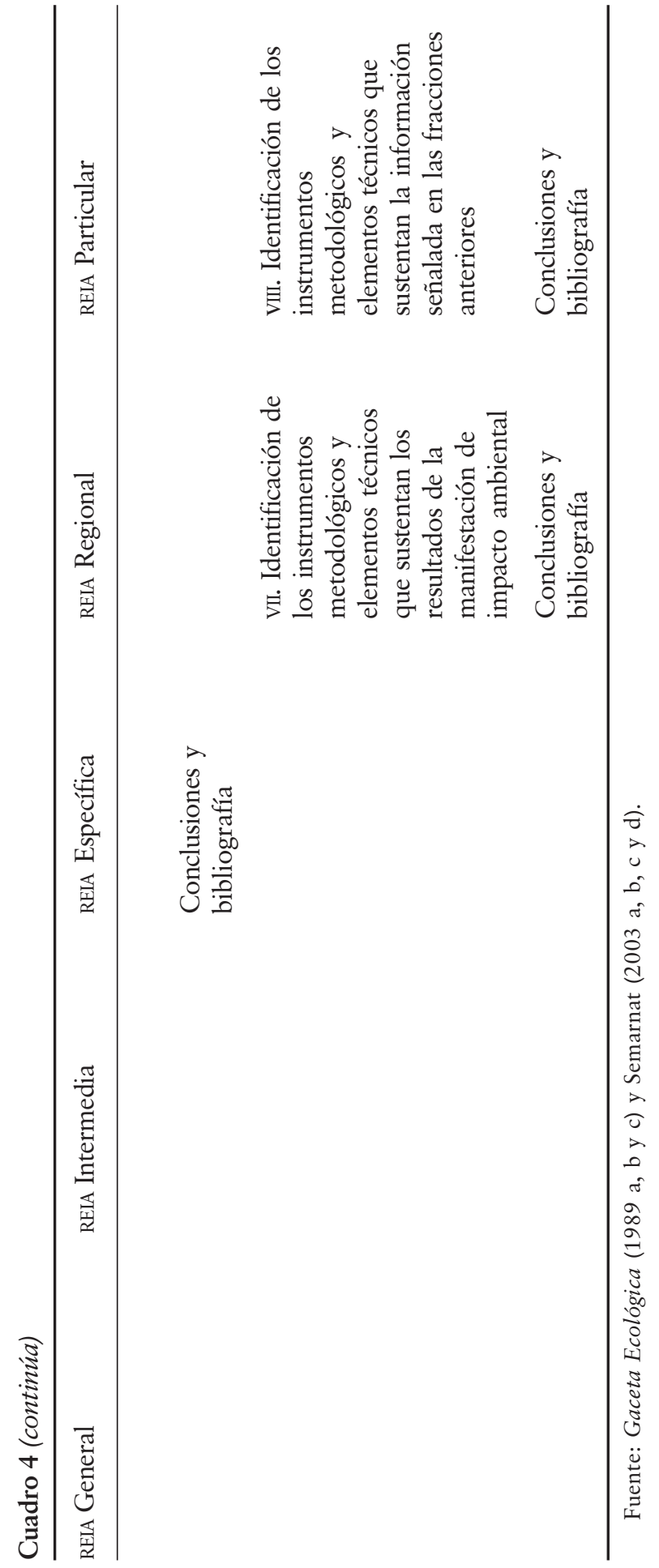




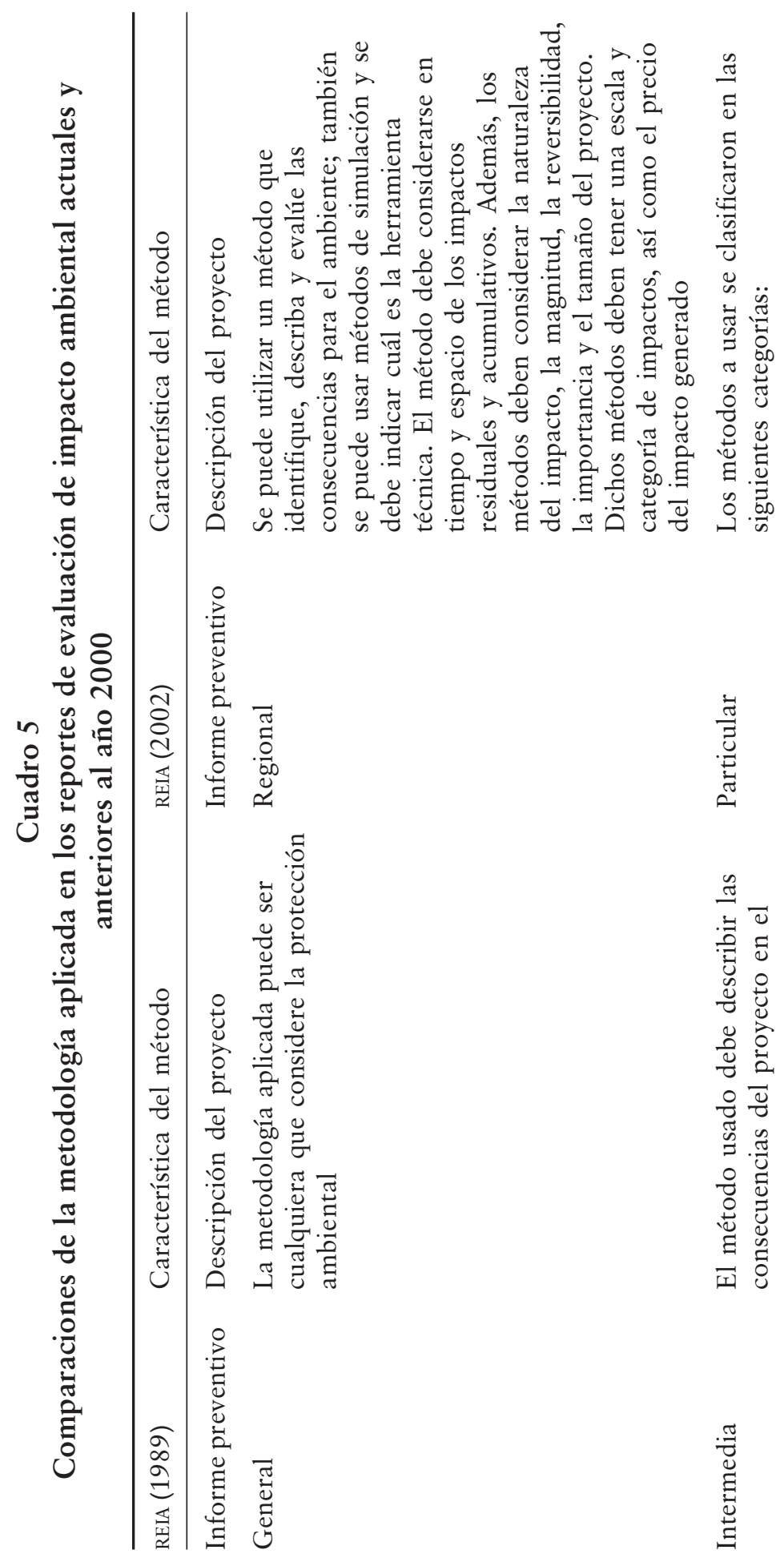




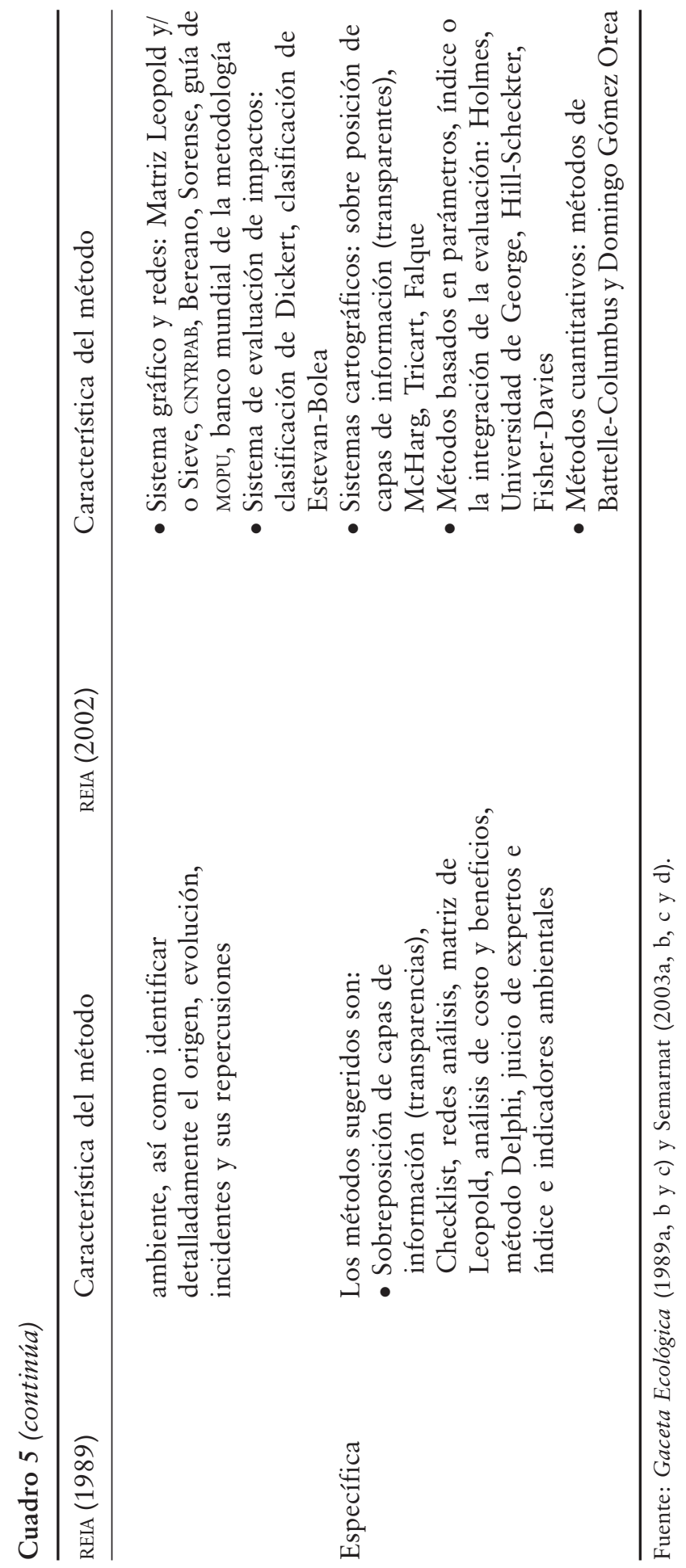


tricos presentaron la siguiente frecuencia relativa en cuanto al uso de métodos:

- $48.8 \%$ de los proyectos empleó el método de Matriz Leopold y/o Sieve y Checklist.

- $17.6 \%$ utilizó cualquier otro método sugerido o conocido por el consultor.

- 14.4\% aplicó algún tipo de matrices modificadas o reducidas, o superposición de transparencias o escenarios modificados.

- $7.2 \%$ usó una descripción personal.

- 6.4\% aplicó diagramas de flujo (Odum, 1972), modelos matemáticos (Conama, 1994: 1) o modelos de dispersión de la contaminación.

Los 15 REIA restantes, que comprendían proyectos hidroeléctricos, geoeléctricos y eoloeléctricos, presentaron las siguientes frecuencias relativas:

- $85.1 \%$ de los proyectos eléctricos aplicó un método descriptivo, Checklist, matrices de Leopold y/o Sieve.

- 14.9\% de los proyectos usó en su evaluación escenarios modificados y el método Coneza-Vitola (Coneza, 1995: 390).

La mayoría de los evaluadores de proyectos eléctricos optan por metodologías tradicionales que resultan de fácil aplicación, bajo costo y corto tiempo de evaluación, pero que desafortunadamente son altamente subjetivas y en muchos casos no evalúan todas las etapas del proyecto.

\subsection{REIA y el sector eléctrico de la región norte de México} 4.2.1. Aspectos socioeconómicos

Un proyecto sostenible es aquel que permite la coexistencia de la parte económica, la social y la ambiental de manera armónica. La EIA y los REIA consideran secundariamente las partes social y económica, ya que el punto central de la evaluación son los aspectos ambientales. Es indispensable evaluar esta parte socioeconómica por la estrecha relación de las actividades productivas y el ambiente. En el caso de la región norte de México, los proyectos o actividades eléctricas tienen gran importancia no sólo por el número de centrales (77 proyectos eléctricos, que generan 6,764.73 GW y representan $37.8 \%$ de la energía eléctrica nacio- 
nal, y 16 proyectos en proceso de factibilidad) (véase cuadro 6), sino además por su relación directa e indirecta con las actividades socioeconómicas que requieren de energía (véase cuadro 7).

La región norte del país se caracteriza sobre todo por su dinámica actividad industrial, que está relacionada con las ramas metálicas básicas, en especial la automotriz, la metálica y la alimentaria, en gran medida exportadoras y crecientes en la modalidad de la maquila. Pero también por las actividades agrícolas y pesqueras orientadas a la exportación, y por sus flujos de importación y exportación vía portuaria, que generan alrededor de $27 \%$ del PIB nacional (Cordera, 2004). Sin duda, todas esas actividades requieren de energía eléctrica para su desarrollo; tal es el caso de la industria manufacturera y del comercio, que, en ese orden, son las actividades más importantes de la zona norte, donde vive $27 \%$ de la población total y $21.98 \%$ de la población ocupada del país, según lo reportado en el año 2000 (Cordera, 2004: 1, 2). En la gráfica II se representa el crecimiento en cuanto a consumo de energía, el crecimiento poblacional y el del PIB, que han tenido una trayectoria similar (véase gráfica II). En el cuadro 8 se representa el PIB producido por divisiones económicas, donde des-

\section{Gráfica II}

Crecimiento de consumo de energía, PIB y población de 1993 a 2003

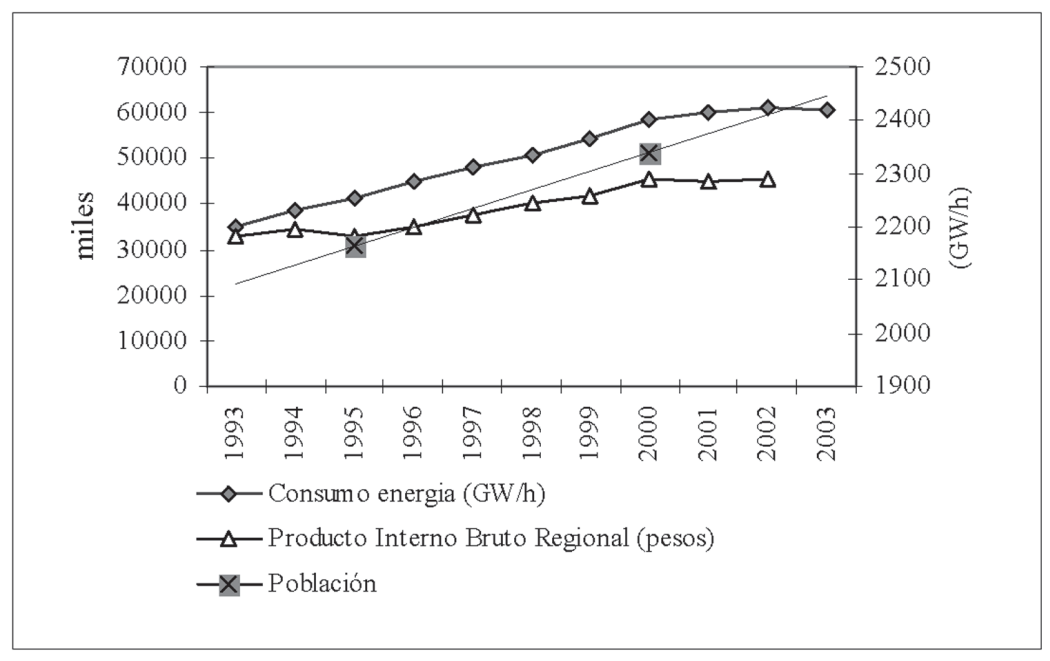

Fuente: Sener (2003), INE (2000) y Conapo (2000a). 


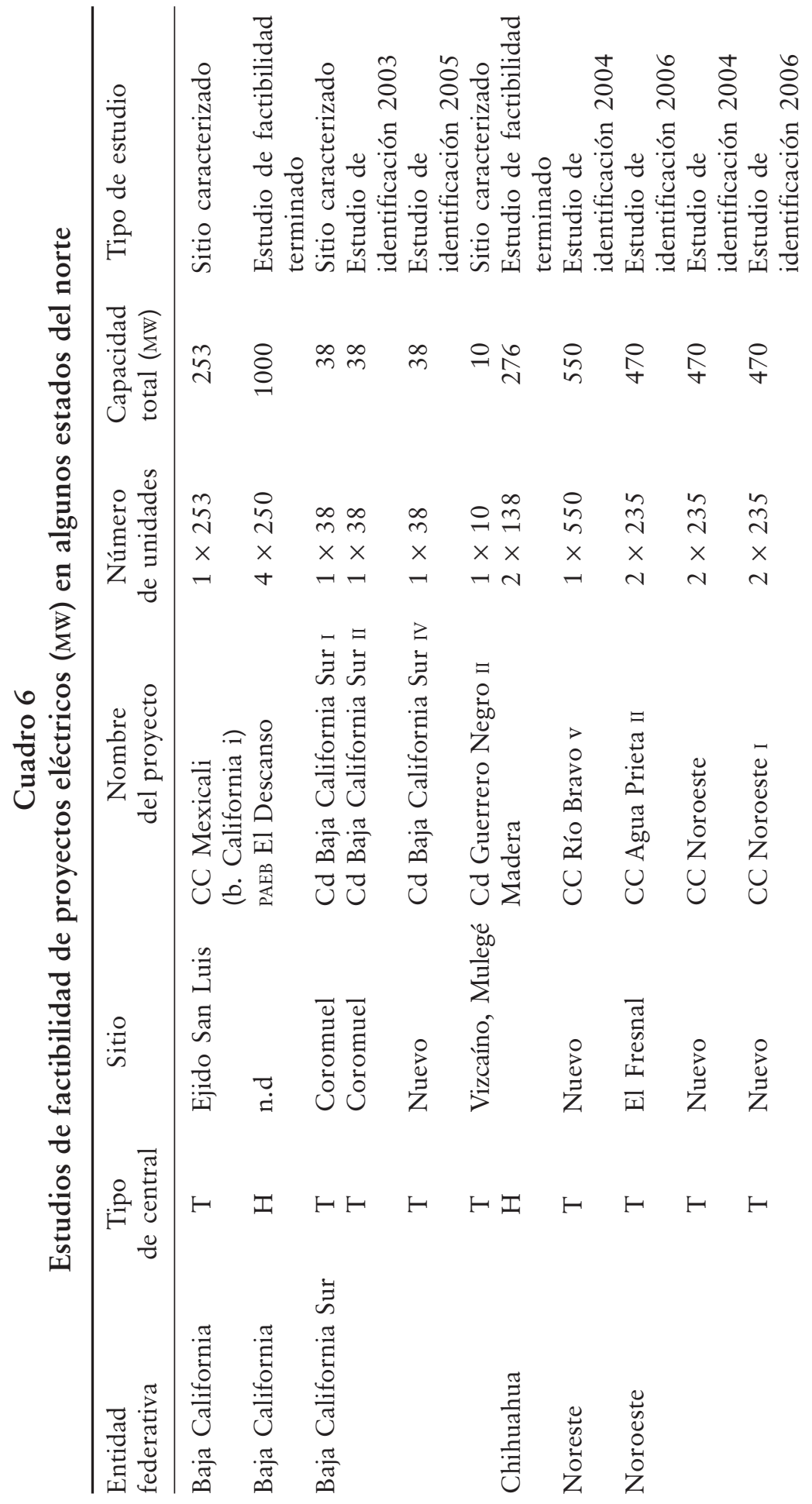




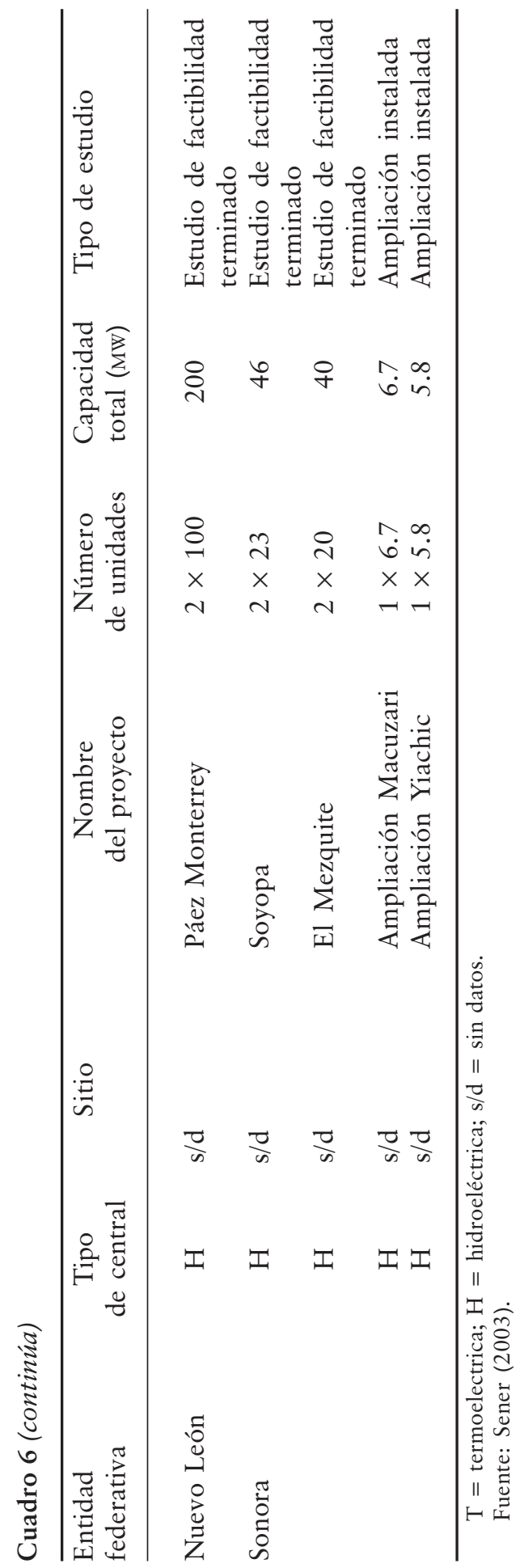




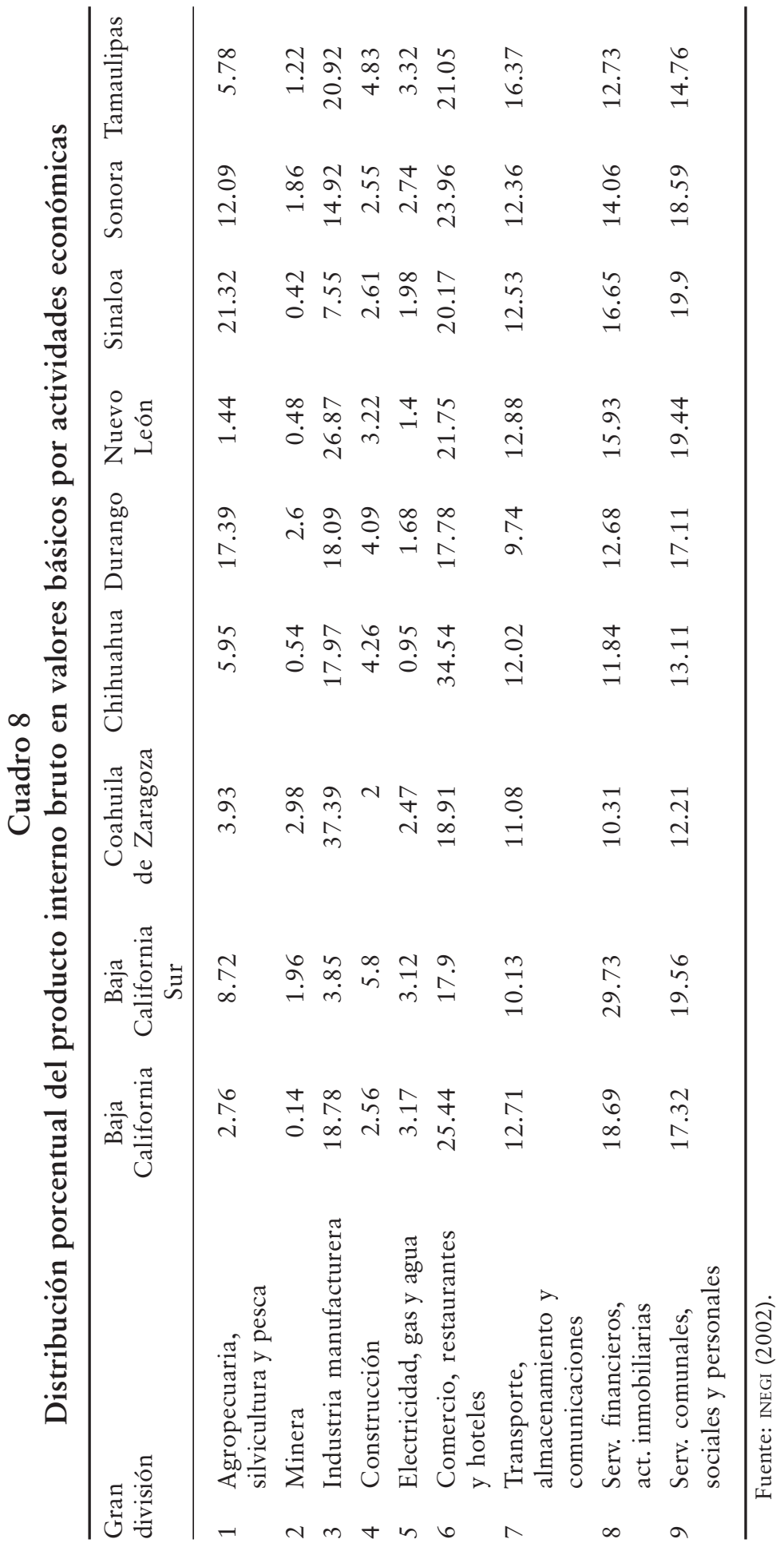




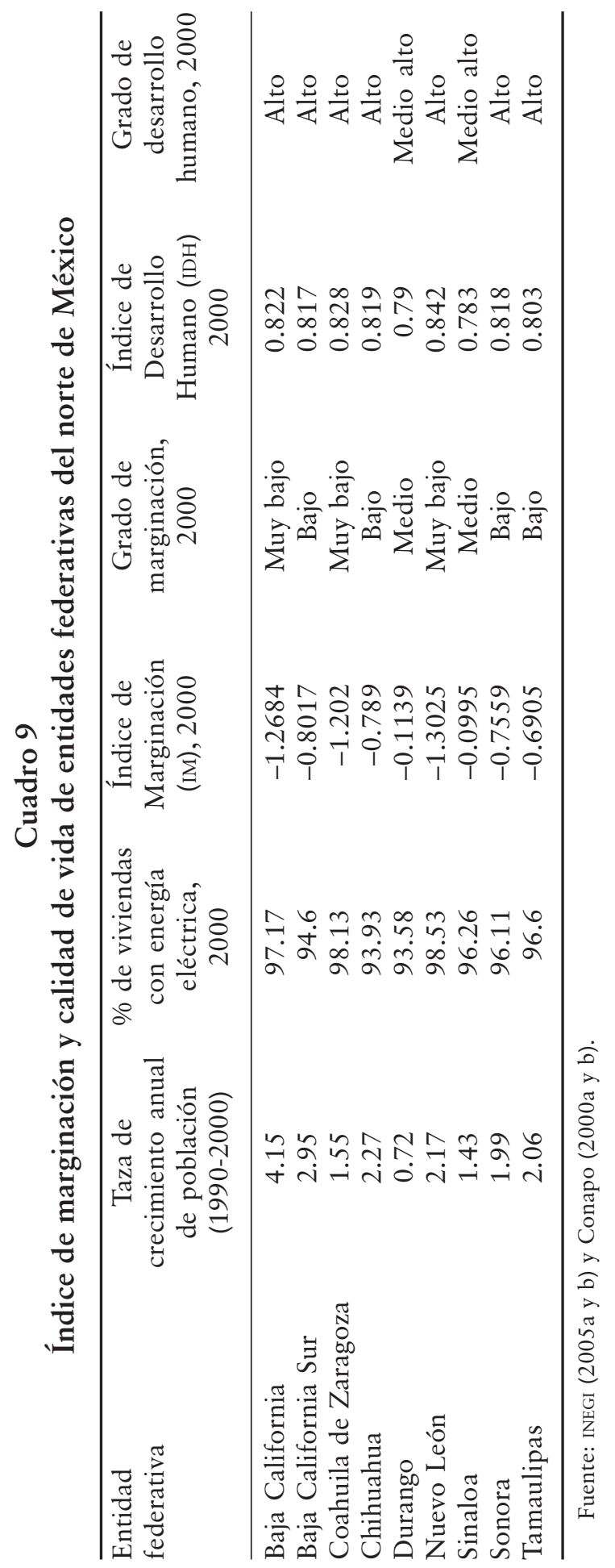


tacan la 3 y la 6 de la región, que comprenden la industria manufacturera y el comercio, restaurantes y hoteles.

En lo social, la electricidad ha beneficiado a 34,934 localidades (INEGI, 2002: 1), lo que representa 27.8\% de las localidades del país, avance que se refleja en un índice de marginación (IM) bajo y en un índice de desarrollo humano (IDH) alto; es decir, en condiciones de vida favorables para esta región en comparación con el resto del país, pero que aún no son óptimas (cuadro 9).

\subsubsection{Aspectos ambientales}

La región norte de país presenta características de desertificación $(53.51 \%$ del total nacional) que demandan realizar correctas evaluaciones de impactos ambientales, sobre todo por el tipo de ecosistemas y procesos edáficos, y particularmente porque esta zona presenta altos valores de erosión hídrica y eólica (INE, 2000: 15). Se deben evaluar asimismo con exactitud los impactos que obras como las eléctricas pueden causar en la diversidad ecológica, ya que la zona cuenta con $29.34 \%$ del total nacional de vegetación boscosa, con $20.69 \%$ de vegetación tipo selva baja caducifolia, y con $72.09 \%$ de vegetación de matorral. En el caso del pastizal, que es importante para actividades ganaderas, representa $26.36 \%$ de total nacional, mientras los cultivos representan $29.83 \%$. En lo que se refiere a superficie con asentamientos humanos, la región cuenta con $31.10 \%$ de total nacional, siendo los estados con más asentamientos Baja California, Nuevo León, Sonora y Tamaulipas.

La diversidad de ambientes en la región implica también una riqueza en flora y fauna (Neyra y Durand, 1998). Asimismo, se tiene la mayor cantidad de áreas protegidas, reservas, santuarios y otros (cuadro 10), que representan $54.77 \%$ de total de la superficie del país. Todo lo anterior nos da un marco de referencia sobre la importancia ambiental de la región y sobre la necesidad de realizar correctas EIA que permitan la coexistencia de la parte social, económica y ambiental. Para ello es necesario evaluar simultáneamente los tres aspectos y englobarlos en proyectos que sean realmente sostenibles, y donde el proceso de la EIA y los REIA resulte en recomendaciones aplicables y útiles que eviten daños futuros. 


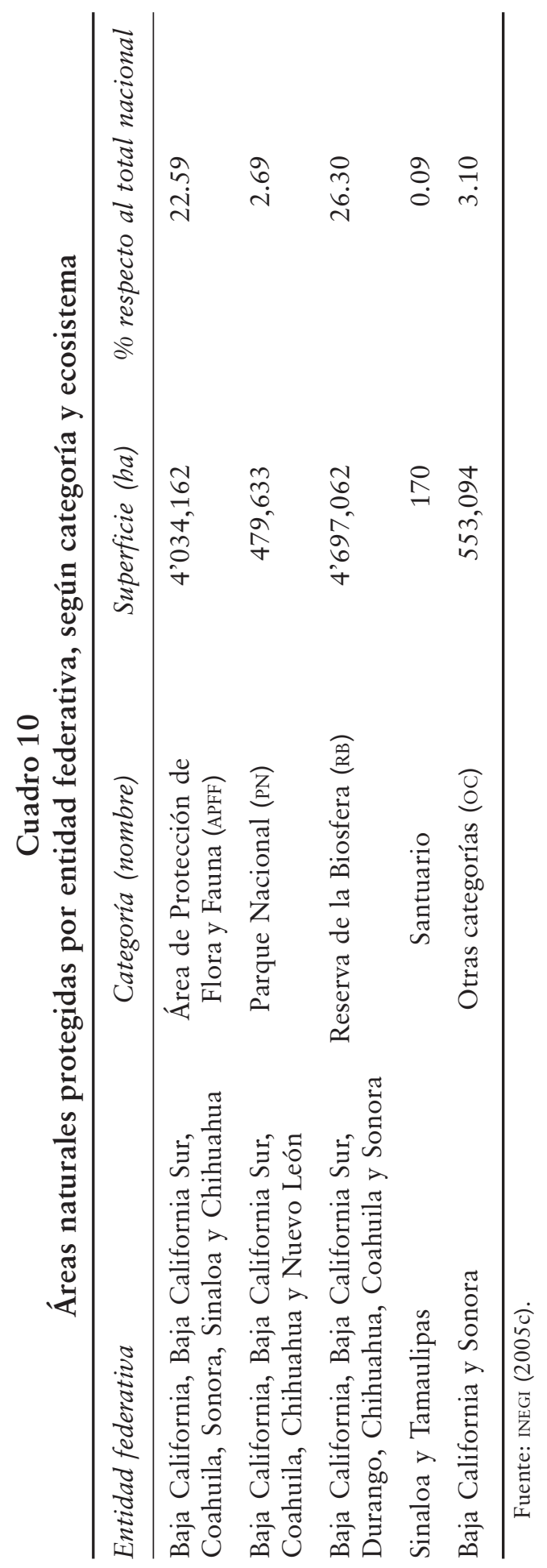




\section{Conclusiones}

Sin duda que se han dado avances en los reportes de evaluaciones ambientales aplicadas a proyectos eléctricos en los últimos 30 años, pero aun con ello se observan criterios heterogéneos, subjetivos y discrecionales en cuanto al procedimiento y tipo de reportes de evaluación, por lo que se hace necesario subsanarlos con una mayor capacitación técnica y actualización tanto de los consultores como del personal de la Semarnat, la CFE y la comunidad científica interesada en este tipo de trabajos. Es indispensable que se creen formatos de evaluación específicos para el sector eléctrico, que consideren todas las actividades propias del sector, y las formas de producción de energía por medios renovables y no renovables. Es asimismo imprescindible que se inicie el desarrollo de metodologías y técnicas de evaluación de impactos ambientales aplicables en las diferentes fases de los proyectos eléctricos, y que se homogeneicen criterios en cuanto a sus regulaciones jurídicas.

Principalmente es indispensable en el futuro inmediato proceder a evaluar y valorar los aspectos económicos y sociales, tan a detalle como actualmente se evalúan los ambientales, para que den como resultado proyectos realmente sostenibles.

\section{Bibliografía}

Ángeles Cornejo, Sarahí (2003), Los efectos de TLCA en los cambios de la organización industrial eléctrica de México, ponencia presentada en el coloquio internacional Energía, Reformas Institucionales y Desarrollo en América Latina, Universidad Nacional Autónoma de México, México. Disponible en: htpp://www.buho.economia.unam.mx/pcientifica/coloquio-erda/11DSarahiAngelesL.pdf, 17 de julio de 2005.

BвvB (Banco Bilbao Vizcaya Bancomer) (2002), Apertura del sector eléctrico, Serie Propuestas, núm. 21, Disponible en: http://www.bancomer.com.mx/economica/prop/ electrico.pdf, 23 de junio de 2004.

Belausteguigoitia, Carlos, Gustavo Merino y Ricardo Samaniego (2001), La inserción de la gestión ambiental en las politicas sectoriales de Latinoamérica y el Caribe: El caso de 
los sectores energético e industrial en México, Centro de Investigaciones en Política Pública, Instituto Tecnológico Autónomo de México, México. Disponible en: http:// www.iadb.org/int/DRP/esp/Red7/Docs/ITAMMexicoAbril4-5-2002.pdf, 27 de agosto de 2004.

BID (Banco Interamericano de Desarrollo) (2001), Revisión de la Evaluación de Impacto Ambiental en Países de América Latina y el Caribe: Metodología, Resultados y Tendencias, Guillermo Espinoza y Viginia Alzina (eds.), Disponible en: http://www.iadb.org/sds/doc/ENVRevEvalImpactoAmbientalLAC-S.PDF, 2 de octubre 2004.

Bojórquez Tapia, Luis A. y Ofelia García (1998), “An Approach for Evaluating EIAs-Deficiencies of EIA in Mexico", Environmental Impact Assessment Review, 18: 217-218, 237.

Breceda Lapeyre, Miguel (2002), Informe para la cooperación ambiental, Programa Medio Ambiente, Economía y Comercio, México, noviembre. Disponible en: htpp:// www.buho.economia.unam.mx/p-cientifica/coloquio-erda/ 16ETeresanchezetaliiLtt.pdf, 13 de julio de 2005.

Campos Aragón, Leticia y Juan Quintanilla Martínez (1997), La apertura externa en el sector eléctrico mexicano, ponencia presentada en el Primer Seminario sobre la Situación y Perspectivas del Sector Eléctrico en México, Instituto de Investigaciones Económicas, Coordinación de Humanidades, Programa Universitario de Energía, Coordinación de Vinculación de la Universidad Nacional Autónoma de México, México. Disponible en: htpp:// www.buho.economia.unam.mx/p-cientifica/coloquio-erda/ 16ETeresanchezetaliiLtt.pdf, 18 de julio de 2005.

CFE (Comisión Federal de Electricidad) (2005), La electricidad en México, http://www.cfe.gob.mx/www2/QueEscfe/informacion/Historia/, 3 de noviembre 2004.

Cibnor (Centro de Investigaciones Biológicas del Noroeste) (1990), Manifestación de impacto ambiental. Modalidad intermedia. Termoeléctrica Puerto San Carlos en Baja California Sur, Centro de Investigaciones Biológicas del No- 
roeste S.A. Consultada en el CIGA, Semarnat, pp. 1, 132, $142,144,145-146$.

Cherp, Oleg y Norman Lee (1997), "Environmental Policy Making: Evolution of SER an OVOS in the Soviet Union and Russia (1985-1996)", Environmental Impact Assessment Review, 17: 177-179; 200-204.

Conama (Comisión Nacional del Medio Ambiental) (1994), Manual de evaluación de impacto ambiental; conceptos y antecedentes básicos, cap. VII "Metodologías de evaluación de impacto ambiental Conama", Santiago de Chile, pp. 104-113. Disponible en: http://www.iadb.org/sds/doc/ ENVFundamentosEvalImpactoAmbiental.pdf, 22 de marzo de 2004.

Conapo (Consejo Nacional de Población) (2000a), Índice de desarrollo humano, cap. 1, p. 21. Disponible en: http:// www.conapo.gob.mx/00cifras/marg2000/005.htm, 2 de marzo de 2005.

(2000b), Índice de marginación por estado, cap. 2, p. 17. Disponible en: http://www.conapo.gob.mx/00cifras/ marg2000/005.htm, 2 de marzo de 2005.

(2000c), Población. Disponible en: http://www.conapo. gob.mx/publicaciones/2000/pdf/3Proydepob.pdf, 2 de marzo de 2005.

Coneza Fernández, Vitora (1995), Guía metodológica para la evaluación del impacto ambiental, Mundi-Prensa, Madrid.

Cordera Campos, Rolando (2004), "Apartado economía y sociedad (Estadísticas Nacionales)", Desigualdad regional y marginación en México. Disponible en: http: \|www.rolando cordera.org.mx, 14 de febrero de 2005.

Ecoprotección Ambiental s.A. de C.v. (1993), Manifestación de impacto ambiental. Modalidad general. Consultado en CIGA, Semarnat, cap. I: 1, 72-75, 96-99; cap. VII: 1-81. 
El-Fadel, Mutasem, Michel Zeinati y Dima Jamali (2000), “EIA Procedure: Framework for Environmental Impact Assessment in Lebanon", Environmental Impact Assessment Review, 20: 579-604.

Ezcurra, Exequiel (1995), "La evaluación de impacto ambiental", Gaceta Ecológica, Instituto Nacional de Ecología (INE), 36: 110. Disponible en: http://www.ine.gob.mx/ueajei/publicaciones/gacetas/gaceta $36 /$ g9536331.html?id_pub=230\&id_tema $=13 \&$ dir $=$ Consultas, 12 de diciembre de 2004.

Gaceta Ecológica (1989a), "Instructivo para la formulación del Informe Preventivo al que los artículos $7^{\circ}$ y $8^{\circ}$ del Reglamento de la Ley General del Equilibrio Ecológico y Protección al Ambiente en materia de Impacto Ambiental", Gaceta Ecológica, Sedesol, I (3): 88. Disponible en: http:/ / ww w. in e.gob.mx/ueajei/publicaciones/ consultaPublicacion.html?id_pub=226, 5 de abril de 2005 .

(1989b), "Instructivo para desarrollar y presentar la Manifestación de Impacto Ambiental en la Modalidad General que se refiere a los artículos $9^{\circ}$ y $10^{\circ}$ del Reglamento de la Ley General del Equilibrio Ecológico y Protección al Ambiente en materia de Impacto Ambiental", Gaceta Ecológica, Sedesol, I, (3): 88. Disponible en:http:// www.ine.gob.mx/u e a jei/publicaciones/ consultaPublicacion.html?id_pub=226, 5 de abril de 2005 .

(1989c), "Instructivo para desarrollar y presentar la Manifestación de Impacto Ambiental en la Modalidad Intermedia que se refiere a los artículos $9^{\circ}, 10^{\circ}$ y $11^{\circ}$ del Reglamento de la Ley General del Equilibrio Ecológico y Protección al Ambiente en materia de Impacto Ambiental", Gaceta Ecológica, Sedesol, I (4): 28. Disponible en: http://www.ine.gob.mx/ueajei/publicaciones/ consultaPublicacion.html?id_pub=226, 5 de abril de 2004 .

(1989d), "Instructivo para desarrollar y presentar la Manifestación de Impacto Ambiental en la Modalidad Específica que se refiere a los artículos $9^{\circ}$ y $12^{\circ}$ del Reglamento de la Ley General del Equilibrio Ecológico y Protección 
al Ambiente en materia de Impacto Ambiental", Gaceta Ecológica, Sedesol, I (4): 38. Disponible en: http:// www.ine.gob.mx/ueajei/publicaciones/consultaPublica cion.html?id_pub $=189 \& i d \_t e m a=\& d i r=C o n s u l t a s \% 3 E \% 3$ Cimg\%20src=, 6 de abril de 2004.

Glasson, John y Salvador Nemesio Neves (2000), "EIA in Brazil: A Procedures-practice Gap. A Comparative Study with Reference to the European Union, and especially the UK", Environmental Impact Assessment Review, 20: 191-225.

Herrera Ordónez, Héctor (2003), "Impacto y riesgo ambiental en México", Memorias del primer encuentro internacional de derecho ambiental, Semarnat, México. Disponible en: http://www.ine.gob.mx/veajei/publicaciones/libros/398/ herrera.html.

Hollick, Malcom (1981), "Environmental Impact Assessment as a Planning Tool," Journal of Environment Management, 12: 79-90.

Hurtado Freyre, Gilberto (1999), La evaluación ambiental de la influencia de la generación eléctrica y disposición de la industria minero metalúrgica, Centro de Tecnología Mineral, Serie Tecnología Mineral 22, Río de Janeiro. Disponible en: http://www.cetem.gov.br/publicacao/ CETEM_STA_22.PDF, 13 de mayo de 2004.

INE (Instituto Nacional de Ecología) (1994), Informe de la situación general en materia de equilibrio ecológico y protección al ambiente. 1993-1994. Reporte Secretaría de Desarrollo Urbano y Ecología-Instituto Nacional de Ecología. México, DF. La evaluación de impacto ambiental: logros y retos, México.

(2000), La evaluación de impacto ambiental. Logros y retos para el desarrollo sustentable 1988-2000, Dirección General de Ordenamiento Ecológico e Impacto Ambiental, INE-Secretaría de Ecología, Medio Ambiente, Recursos Naturales y Pesca, México. 
INEGI (Instituto Nacional de Estadística, Geografía e Informática) (2001), XV Censo industrial 1999. Censos económicos. Sector eléctrico. Disponible en: http://www.inegi.gob.mx/ prod_serv/contenidos/espanol/catalogo/ default.asp? accion $=\& u p c=702825176037 \&$ seccionB $=c p$, 2 de junio de 2005.

(2002), Sistema de cuentas nacionales de México. PIB entidad federativa 1993-2002, Disponible en: http:// www.inegi.gob.mx/prod_serv/contenidos/espanol/bvinegi/ productos/derivada/regionales/pib/pibe1.pdf, 14 de enero de 2005.

(2003), El sector energético en México, INEGI, México, pp. 63-65.

(2005a), Tasa de crecimiento media anual de la población por entidad federativa, 1990 a 2000. Disponible en: http:/ /www.inegi.gob.mx/est/contenidos/espanol/rutinas/ ept.asp? $\mathrm{t}=$ mpob09\&c $=3186,2$ de junio de 2006.

(2005b), Porcentaje de viviendas particulares habitadas por entidad federativa según disponibilidad de sus servicios, 1990 y 2000. Disponible en: http://www.inegi.gob.mx/est/ contenidos/espanol/rutinas/ept.asp? $\mathrm{t}=$ mviv09\&c $=3407,2$ de junio de 2006.

(2005c), Estadísticas ambientales. Áreas naturales protegidas por entidad federativa, según categoría y ecosistema, INegi, México, p. 1. Disponible en: http:// www.inegi.gob.mx/est/contenidos/espanol/rutinas/ ept.asp? $\mathrm{t}=$ mamb05 \&c $=5862,4$ de enero de 2005 .

La sociedad civil, el sector privado y el Estado ante la Evaluación de Impacto Ambiental (2000), Centro de Estudios del Sector Privado para el Desarrollo Sustentable-Unión de Grupos Ambientalistas (IAP)-Centro Mexicano de Derecho Ambiental, México. Disponible en: http:// www.cce.org.mx/cespedes/publicaciones/otras/impactoambiental/contenido.htm. 
Leopold, Luna B., Frank E. Clarke, Brush B. Hanshaw y James R. Balsley (1971), A Procedure for Evaluating Environmental Impact, Government Printing Office, Geological Survey Circular 645, Washington.

Neyra González, Lucila y Leticia Durand Smith (1998), "Biodiversidad", La diversidad biológica de México: estudio de país, cap. 3, parte II, Comisión para el Conocimiento de la Biodiversidad (Conabio), pp. 82-92. Disponible en: http:/ /www.conabio.gob.mx/conocimiento/estrategia_nacional/ doctos/CAP3.PDF, 13 de noviembre de 2004.

Odum, Howard T. (1972), "Use of Energy Diagrams for Environmental Impact Statement”, Tools of Coastal Management, actas de la conferencia, Marine Technology Society, Washington DC, pp. 197-231.

OLADE-BID (Organización Latinoamericana de Energía-Banco Interamericano de Desarrollo) (1994), Guía para la evaluación de impacto ambiental de centrales termoeléctricas, OLADE-BID, Quito, Ecuador.

Pisanty-Levy, Julieta (1976), Aplicación preliminar del método de evaluación de impacto ambiental ocasionado por la planta núcleo eléctrica de Laguna Verde, Veracruz, tesis de licenciatura en biología, Facultad de Ciencias, Universidad Nacional Autónoma de México, México.

(1993), "México Environmental Assessment Experience”, Environmental Impact Assessment Review, 13 (6): 267272.

Purnama, Dadang (2003), "Reforms of the EIA Process in Indonesia: Improving the Role of Public Involvement," Environmental Impact Assessment Review, 23: 413-428, 434-439.

Rodríguez Padilla, Víctor (1999), Impacto de la reforma económica sobre las inversiones de la industria eléctrica en México: el regreso del capital privado como palanca del desarrollo, Naciones Unidas-Comisión Económica para 
América Latina y el Caribe, Serie: Reformas económicas, núm.18, Santiago de Chile.

Sánchez Salazar, María Teresa, José María Casado Izquierdo y Eva Saavedra Silva (2005), La inversión privada en el sector eléctrico en México: antecedentes, características y estructura territorial, Instituto de Geografía de la Universidad Nacional Autónoma de México, México. Disponible en: htpp://www.buho.economia.unam.mx/p-cientifica/ coloquio-erda/16ETeresanchezetaliiLtt.pdf, 15 de julio de 2005.

Semarnat (Secretaría de Medio Ambiente y Recursos Naturales, (2002), Compendio de Estadísticas Ambientales, Semarnat, México. Disponible en: http://www.semarnat.gob.mx/ estadisticas_2000/Presentacion/index.shtml, 22 de enero de 2005.

(2003a), Guía para elaborar la manifestación de impacto ambiental modalidad regional de proyectos de generación, transmisión y transformación de energía eléctrica, http:// portal.semarnat.gob.mx/semarnat/portal/!ut/p/kcxml/ 04_Sj9SPykssy0xPLMnMz0vM0Y_QjzKLN4g3NA4ESYGY xqb6kWhCjhgivoYQIV8jmEgAVCTADCYS5gkRCfOCiRga W8BMskSIecDEPBFiAQgbfT3yc1P1QxGOMzTVjzDT99Y P0C_IDYWAiPIKE0dFRQDDzFLv/delta/base 64xml/ L3dJdyEvd0ZNQUFzQUMvNEIVRS82XzBfMTNQ, 27 de julio de 2005 .

(2003b), Guía para elaborar la manifestación de impacto ambiental modalidad particular de proyectos de generación, transmisión y transformación de energía eléctrica, http://portal.semarnat.gob.mx/semarnat/portal/!ut/p/ kcxml04_Sj9SPykssy0xPLMnMz0vM0Y_QjzKLN4g3NA 4ESYGYxqb6kWhCjhgivoYQIV8jmEgAVCTADCYS5gkRCf OCiRgaW8BMskSIecDEPBFiAQgbfT3yc1P1QxGOMzTVj zDT99YP0C_IDYWAiPIKE0dFRQDDzFLv/delta/ base64xmlL3dJdyEvd0ZNQUFzQUMvNEIVRS82XzBfMTNQ, 28 de julio de 2004.

(2003c), Guías Sectoriales para la elaboración del Informe Preventivo, MIA Particular y MIA Regional. Disponible 
en: http://portal.semarnat.gob.mx/semarnat/portal/!ut/p/ kcxml04_Sj9SPykssy0xPLMnMz0vM0Y_QjzKLN4g3NA4ESYG Yxqb6kWhCjhgivoYQIV8jmEgAVCTĀDCYS5gkRCfOCiRga W8BMskSIecDEPBFiAQgbfT3yc1P1QxGOMzTVjzDT99YP 0 C _ I D Y W A i P I K E 0 d F R Q D D z F L v / deltabase64xmlL3dJdyEvd0ZNQUFzQUMvNEIVRS82XzBfM TNQ, 2 de noviembre de 2004.

(2003d), Antecedentes de la Semarnat. Disponible en: http://www.semarnat.gob.mx/portal/queessemarnat/ Antecedentes.htm, 2 de noviembre de 2004.

(2006), Dirección de Riesgo e Impacto Ambiental (Consulta el estado actual de tu trámite), 51 volúmenes consultados de 1988 a 2004. Disponible en: http:// portal.semarnat.gob.mx/semarnat/portal/!ut/p/kcxml/ 04_Sj9SPykssy0xPLMnMz0vM0Y_QjzKLN4g3NA4E SYGYxqb6kWhCjhgivoYQIV8jmEgAVCTADCYS5 gk RCfOCiRgaW8BMskSlecDEPBFiAQgbfTyc1P1QxGOMzTVzDT 99YP0C_IDYWAiPIKE0dFRQDDzFLv/delta/base64xml/ L3dJdyEvd0ZNQUFzQUMvNEIVRS82XzBfMTNQ, 1 de junio de 2006, o consulta directa en Semarnat, Av. Revolución 1425, col. Tlacopac San Ángel, delegación Álvaro Obregón, CP 01040, México, DF.

Sener, (Secretaría de Energía) (1999), Breve cronología del sector eléctrico de energía, http://www.energia.gob.mx/ frame7.html, 13 de enero de 2004.

(2003), Prospectivas del sector eléctrico 2003-2012, Sener, México.

Recibido: 3 de mayo de 2005. Reenviado: 15 de agosto de 2005. Aceptado: 29 de agosto de 2005.

María E. González Ávila es maestra en ciencias (Sistemas y Recursos Acuáticos) por la UNAM; candidata a doctora en ciencias (Uso y Manejo de los Recursos Naturales) en el Centro de Investigaciones Biológicas del Noroeste, La Paz, Baja California Sur. Ha participado en cursos y pláticas. Tiene experiencia en con- 
sultoría y docencia ambiental desde 1999 a la fecha. Entre sus publicaciones están: “On the Influence of Hot-water Discharge on Plankton Communities from Coastal Zone of the Gulf of Mexico", Water, Air and Soil Pollution 119: 209-230, del 2000; "Potencial de aprovechamiento de la energía eólica para la generación de energía eléctrica en zonas rurales de México", Interciencia, 31 (4), abril de 2006; "Environmental Impact Assessment for Alternative-energy Power Plants in Mexico", Journal Environmental Biology (aceptado para su publicación en agosto 2005).

Luis Felipe Beltrán Morales. Es licenciado en economía por la Universidad Autónoma de Baja California Sur (UABCS), México; doctor en ciencias ambientales por el Centro EULA-Chile; investigador del Centro de Investigaciones Biológicas del Noroeste, Cibnor, s.c.; profesor de la Maestría en Economía del Medio Ambiente y Recursos Naturales de la UABCS, y actualmente director de Estudios Ambientales del Cibnor, s.c. Publicaciones recientes: "Valoración socioambiental de los recursos naturales: el caso de los recursos minerales en la parte central de Baja California Sur, México", Boletín del Instituto de Geografía, UnAM, 57: 8194 (2005); "Environmental and Socioeconomic Multivariate Analysis of the Primary Economic Sector of Mexico", Sustainable Development, 11 (2): 77-83 (2003); "Relaciones socioambientales en el ámbito rural de la porción central de Baja California Sur, México", Boll. Geogr. Apl. 4: 54-80 (2003); "Economic and Social Effects Caused by NAFTA in the Fisheries of Tuna in Baja California Sur, Mexico", Problemas del Desarrollo, Revista Latinoamericana de Economía 33 (129): $43-53$ (2002), y "Apertura comercial y medio ambiente", Interciencia, 27 (5): 259-263 (2002). Es miembro del Sistema Nacional de Investigadores nivel I.

Julio César Peralta Gallegos tiene la maestría en ciencias (Uso y Manejo de Recursos Naturales) en el Cibnor, y está por obtener el doctorado en ciencias con el mismo perfil y en la misma institución. Dedicó 17 años de su experiencia profesional a trabajos relacionados con ecosistemas de ambientes hipersalinos relacionados con la producción y calidad de la sal común $(\mathrm{NaC})$. Sus tesis profesionales de licenciatura y maestría se relacionan con aspectos de la avifauna en esos ambientes. Ha publicado el artículo científico Predation by the Western Gull on the Pared Grebe 
at a Salina in Mexico. Actualmente es delegado federal de la Procuraduría Federal de Protección al Ambiente (Profepa) en el estado de Baja California Sur.

Enrique Troyo Diéguez. Es doctor en ciencias por la UNAM; investigador nacional nivel II del Sistema Nacional de Investigadores de México, y miembro de la Academia Mexicana de Ciencias. Se desempeña como investigador titular y catedrático del Programa de Posgrado en el Cibnor, en las líneas de investigación sobre uso y conservación del agua y recursos naturales en zonas áridas, con cerca de 70 publicaciones científicas en revistas nacionales e internacionales. Es miembro de la Sociedad Mexicana de la Ciencia del Suelo y de la American Geophyscal Union.

Alfredo Ortega Rubio es doctor en ciencias con especialidad en ecología por el Instituto Politécnico Nacional. Premio Nacional Reconocimiento a la Conservación de la Naturaleza 2003, categoría Académica y de Investigación. Premio Nacional al Mérito Nacional Forestal y de la Vida Silvestre 1993. Actualmente es investigador nacional nivel III del Sistema Nacional de Investigadores de la República Mexicana. Es autor y coautor de 125 artículos de investigación original. Ha editado 13 libros y publicado 45 capítulos de libros. Ha dirigido 78 proyectos de restauración ecológica e impacto ambiental; además, ha dirigido 34 proyectos de manejo de recursos, entre ellos los que permitieron instaurar y desarrollar las reservas de la Biosfera de Sierra de la Laguna, Desierto del Vizcaíno e Islas Revillagigedo. 